\title{
Stereochemistry of transient protein-protein interactions in a signaling hub: exploring G3BP1-mediated regulation of CFTR deubiquitination
}

\author{
Colin T. Sheehan and Dean R. Madden*
}

Department of Biochemistry and Cell Biology, Geisel School of Medicine at Dartmouth, Hanover, NH, USA

*Corresponding author. 7200 Vail Building, Room 408, Hanover, NH, 03755-3844, USA. E-mail address: dean.r.madden@dartmouth.edu.

Keywords: intrinsically disordered protein, protein-protein interaction, protein complex, peptide interaction, protein structure, crystal structure, crystallography, mutagenesis, mutagenesis in vitro, deubiquitination, bacterial pathogenesis, neurodegeneration, cancer 


\section{Abstract}

Intrinsically disordered proteins (IDPs) can coordinate often transient or weak interactions with multiple proteins to mediate complex signals within large, reversible protein networks. Among these, the IDP hub protein G3BP1 forms protein complexes with Caprin1 and USP10, and the resulting control of USP10 activity plays an important role in a pathogenic virulence system that targets CFTR endocytic recycling. However, while the identities of protein interactors are known for many of these IDP hub proteins, the relationship between pairwise affinities and the extent of protein recruitment and activity is not well understood. Here we describe in vitro analysis of the G3BP1 affinities, and show that substitution of G3BP1 residues F15 or F33 to tryptophan reduces affinity for both the USP10 and Caprin1 motif peptides. These same mutations significantly reduce formation of complexes by the full-length proteins. The crystal structure of G3BP1 TripleW (F15W/F33W/F124W) mutant reveals a clear reorientation of the side chain of W33, creating a steric clash with the USP10 and Caprin1 peptides. An amino-acid scan of the USP10 and Caprin1 motif peptides reveals similarities and differences in the ability to substitute residues in the core motifs as well as specific mutations with the potential to create higher affinity peptides. Taken together, these data show that small changes in 1:1 binding affinity can have significant effects on the composition of cellular interaction hubs. These specific protein mutations can be harnessed to manipulate complex protein networks, informing future investigations into roles of these networks in cellular processes. 


\section{Introduction}

Intrinsically disordered proteins (IDPs) are important molecular machines that play critical roles in cellular processes and signaling pathways $(1,2)$. Such proteins lack a welldefined three-dimensional globular structure and can range from fully to partially unstructured. This stereochemical flexibility allows these proteins to interact promiscuously with different proteins in a context-dependent manner (3). Additionally, these proteins often utilize conserved sequence motifs to facilitate protein-protein interactions with high specificity and modest affinity leading to rapid signal transduction (4-6). IDPs often function as interaction hubs as well as facilitators of biomolecular condensation. Ras GTPase Activating Protein SH3 Domain Binding Protein 1 (G3BP1), a protein containing many intrinsically disordered regions, is reported to serve as an interaction hub in stress granules (SG) and to form transient interactions with USP10 and Caprin1, which in turn influence the SG network organization and signaling (7-9).

G3BP1 is a $52 \mathrm{kDa}$ protein ubiquitously expressed in the cytoplasm. Current research supports the theory that the main role of G3BP1 is to triage mRNA in response to intracellular and extracellular stimuli. However, significant evidence suggests that G3BP1 plays a central role in several additional cellular processes, including rasGAP signaling, ubiquitination, mRNA metabolism, and stress granule (SG) formation, and these diverse functions appear to be modulated by G3BP1's interaction with RNA and other proteins (10-18). The large protein network surrounding G3BP1 has been implicated in several diseases including neurological and neurodegenerative disorders, cancer progression, bacterial pathogenesis, and viral infection $(14,16,17,19,20)$. The 
ability to selectively modulate protein-protein interactions within this large, intricate protein network would represent a novel drug target with far reaching implications (21-24).

G3BP1 and the other two members of the G3BP family all share the following five domains: an N-terminal nuclear transport factor 2-like domain (NTF2), a central region consisting of several proline rich (PxxP) motifs, an acid-rich domain, a canonical RNA recognition motif $(\mathrm{RRM})$, and a loosely conserved $\mathrm{C}$-terminal arginine and glycine rich (RGG) box (25). Except for the NTF2-like domain and RNA-binding domain, G3BP proteins are predicted to be largely disordered. A recently reported model suggests that interactions with RNA and proteins partners modulate the structure and provide relative stability to G3BP1 $(8,9)$.

The NTF2 domain is most highly conserved domain in the G3BP family and plays a role in several G3BP functions including dimerization, protein binding, and SG formation $(13,26,27)$. Proteins known to interact with G3BP1 NTF2 include rasGAP, Caprin1, and USP10 $(10,15,28)$. Multiple groups have shown that Caprin1 and USP10 compete for the same binding groove on the G3BP1 NTF2 domain $(7,8,29)$. Recent G3BP1 NTF2 co-crystal structures have identified three phenylalanine residues (F15, F33, and F124) that are responsible for coordinating peptides from Caprin1 and USP10 (26, 30). Additionally, several groups have shown that mutating F33 to a tryptophan ablates Caprin1 and USP10 binding $(8,26,31,32)$.

Ubiquitin Specific Peptidase 10 (USP10) is an $87 \mathrm{kDa}$ cysteine protease and a member of the deubiquitinating enzyme (DUB) family. As a DUB family member, USP10 plays an important role in protein homeostasis and has been shown to remove conjugated ubiquitin from targets like p53/TP53, BECN1, SNX3 and the cystic fibrosis 
transmembrane conductance regulator (CFTR) (33-36). Additionally, USP10 has been implicated in a variety of diseases including cancer, where it can act as an oncogene or a tumor suppressor, as well as Alzheimer's disease and other neurodegenerative diseases $(19,37-40)$.

USP10 contains a highly conserved catalytic domain (USP domain) beginning at residue R415 and spanning most of the C-terminal end of the protein. Similar to G3BP, the $\mathrm{N}$-terminal half of the protein is predicted to be mostly disordered. First identified as a G3BP-interacting protein in a yeast two-hybrid screen, subsequent reports have identified a core motif (FGDF; residues 10-13) in USP10 that is recognized by G3BP1 NTF2 $(15,29)$. A co-crystal structure of NTF2 complexed with FGDF-containing peptides revealed that both motif phenylalanine residues (F10 and F13) protrude into the NTF2 binding grove and form $\pi$-stacking interactions with NTF2 residues F15, F33, and F124 $(26,32)$. Alanine substitution of USP10 F10, G11, or F13 completely ablates G3BP1 binding, while D12A allows for weak binding (32). These data show that the FGDF is necessary and sufficient for G3BP1 binding.

Cytoplasmic Activating/Proliferation Associated Protein 1 (Caprin1) is a $78 \mathrm{kDa}$ cytoplasmic RNA-binding protein. Caprin1 has been implicated in cell-cycle regulation and cell proliferation where it acts alone or in combination with other RNA-binding proteins such as G3BP1 and fragile X mental retardation protein (FMRP) (28, 41-44). Caprin1 binds RNA via its C-terminal RNA-binding RGG motif and an RG enrichment region (28). Caprin1 contains a highly conserved $F(M / I / L) Q(D / E) S z(I / L) D$ motif spanning residues 372-379 that is recognized by G3BP1 NTF2 (28). A recent co-crystal structure revealed that the Caprin1 $\mathrm{YNFI}(\mathrm{Q})$ segment binds in the same hydrophobic grove on NTF2 as the 
USP10 FGDF motif (30). The structure further highlighted the important role of NTF2 residues F15, F33, and F124 in coordinating Caprin1 and USP10 peptides.

G3BP1 and its interactions with USP10 and Caprin1 are important for SG formation and regulation. Additionally, USP10 and Caprin1 have distinct roles in several cellular processes separate from G3BP1 and SGs $(28,33,34,45,46)$. However, there is limited information on the importance of specific conserved motifs for the stability of IDP interactions. We therefore sought to better understand the interaction of these proteins at the residue level and to generate better tools for future investigations. We report here the first crystal structure of G3BP1 NTF2 harboring three phenylalanine substitutions in residues F15, F33, and F124 (TripleW). Additionally, we show a full amino-acid scan of the USP10 and Caprin1 binding motif peptides and correlate these results with cellular protein association via co-purification assays.

\section{Results}

\section{G3BP1 phenylalanine residues contribute differentially to USP10 binding}

The highly conserved G3BP1 NTF2 domain binds peptide motifs from USP10 (15, $28,29)$. To further understand the contribution of specific residues in the G3BP1 binding groove to the interaction, we established a binding assay using fluorescence polarization. The G3BP1 NTF2 domain spanning residues 1-139 was recombinantly expressed and purified from E. coli. Using a USP10-derived octameric reporter peptide ( $F^{*}$-YIFGDFSP; $F^{*}$, fluorescein-aminohexanoic acid tag) containing the core FGDF motif, a binding isotherm was determined for WT-NTF2 (Figure 1A). The experimental isotherm was fit by non-linear least-squares to a single-site binding curve, yielding an equilibrium dissociation 
constant $\left(K_{\mathrm{D}}\right)$ of $3.8 \mu \mathrm{M}$. This value is two-fold stronger than previously reported NTF2 KD value of $7 \mu \mathrm{M}$ for an FGDF-containing nsP3 peptide (32), and 30-fold stronger than the $115 \mu \mathrm{M}$ value reported for a DSGFSFGSK peptide (27). Having established a robust and repeatable assay using several different batches of NTF2, we next sought to test the effects of mutation on binding affinity. Residues F15, F33, and F124 in NTF2 are reported to play a role in coordinating the FGDF peptide from USP10 $(17,26,32)$, so we focused our investigation on these residues, mutating resides F15, F33, and F124 to tryptophan $(29,32)$, singly and in combinations. Additional constructs were created to test other amino-acid mutations for these three residues, including alanine and tyrosine, but most of these constructs had low expression or produced insoluble protein when introduced in combination, and thus were not included in our analyses. Substitutions of tryptophan for F15 or F33 yielded 3.5- and 7.5-fold decreases in affinity, respectively (Table 1). By itself, the F124W mutant caused no significant change in binding affinity. This agrees with multiple reports that G3BP1 harboring a F33W mutation is deficient in USP10 complex formation, whereas F124W has no effect on complex formation $(26,31,32)$

Next we created double and triple mutants to see if the effects of the single substitutions were additive. NTF2 F15/33W and F33/124W exhibited significant increases in $K_{\mathrm{D}}$ values compared to either of the component single mutants. However, NTF2 F15/124W was only modestly worse than F15W alone. The most potent mutant was NTF2 TripleW with a $K_{D}$ of $172.3 \mu \mathrm{M}$, although it was only modestly worse than $\mathrm{F} 15 / 33 \mathrm{~W}$. These data suggest that the contribution of F124 to USP10 peptide binding is much less than might be expected based on earlier co-crystal structures $(17,26)$. Comparison of the F33/124W double mutant to the F33W single mutant reveals a potential context- 
dependent contribution of $\mathrm{F} 124 \mathrm{~W}$ in the presence of F33W. However, comparison of TripleW vs. F15/33W shows that this role of $\mathrm{F} 124 \mathrm{~W}$ is largely lost in the presence of F15W.

Our FP experiments revealed that we could manipulate the equilibrium dissociation constant of NTF2 and USP10 peptide across almost two orders of magnitude using specific tryptophan substitutions. However, we also wanted to test the effect these mutations would have on the ability of full-length versions of G3BP1 and USP10 to interact and form complexes in a cellular context. We overexpressed His-tagged full-length G3BP1 in Expi293 cells, performed immobilized metal-affinity purification, and noticed that the same molecular weight bands were co-purifying across many optimization trials (Supplementary Figure 1). We performed Western Blot analysis on the first fraction and determined that USP10 and Caprin1 were co-purifying with G3BP1 (Figure 1B). We decided to use this overexpression and affinity capture system to assay for binding stability of G3BP1 mutants. Substitution of tryptophan for F33 caused a $90 \%$ reduction in USP10 co-purification as compared to WT (Figure 1C). Any double and triple mutant harboring a F33W mutation (F15/33W, F33/124W, and TripleW) had a similar reduction in USP10 binding ability as compared to F33W alone. F15W alone caused a $\sim 4 \%$ reduction in USP10 co-purification, and when combined with F124 substitution in the F15/124W the reduction was increased to $\sim 53 \%$. The F124W single mutant displayed a modest increase in USP10 co-purification as compared to WT. Since F124W does not significantly disrupt the equilibrium dissociation constant for USP10 as seen in the FP assay, overexpression of full length F124W should retain the full ability to bind and interact with USP10. When the results from these separate experiments are compared, it is 
evident that changes in binding affinity of G3BP1 NTF2 domains for the USP10 FGDF peptide correlate with changes in full length G3BP1:USP10 complex stability (Figure 2). Residues F15, F33, and F124 each help to stabilize the interaction with USP10, but with significantly different contributions.

\section{Caprin1 is more sensitive than USP10 to G3BP1 NTF2 modifications}

Caprin1 competes with USP10 in binding the NTF2 domain, however Caprin1 lacks an FGDF motif (29). Solomon et al. reported that Caprin1 contains a conserved binding motif, FIQDSMLD, spanning residues 372-379 (28). Given the differences in peptide recognition sequences, we wanted to determine whether the three phenylalanine residues (F15, F33, and F124) identified as USP10 modulators also participate in binding and coordinating the Caprin1 peptide. We created a Caprin1-derived dodecamer reporter peptide ( $F^{*}$-YNFIQDSMLDFE) to be used in FP assays. Using recombinant NTF2 and the Caprin1 reporter peptide, we generated a binding isotherm that could be fit to a singlesite binding curve, yielding an estimate of the equilibrium dissociation constant of $1.7 \mu \mathrm{M}$ (Figure 3A). This is approximately 2.2-fold stronger than the $K_{\mathrm{D}}$ calculated for USP10, which disagrees with a recent publication from Schulte et al. that reported a stronger interaction between USP10:NTF2 than between Caprin1:NTF2 in ITC experiments (30). One possible explanation for this discrepancy is the $\mathrm{pH}$ : Schulte et al used $\mathrm{pH} 7.5$, whereas we used $\mathrm{pH} 8.5$ in our experiments. G3BP1 residues $\mathrm{H} 31$ and $\mathrm{H} 62$ have been implicated in Caprin1 binding and have predicted $p K_{a}$-values of 6.1 and 6.2, respectively. Depending on the local pKa of the histidine side chains, they could be differentially protonated at $\mathrm{pH} 7.5$ or $\mathrm{pH}$ 8.5. Alternatively, this discrepancy could be explained by the 
construct design, as the ITC experiments used longer ( 30 aa) USP10 and Caprin1 peptides. If correct, this suggests that residues outside of canonical binding motifs may also contribute to these protein interactions. However, the affinities of the longer peptides are not substantially different from those observed with our much shorter motif peptides, suggesting that the extent of modulation by flanking residues is relatively modest.

Mutation of F15 or F33 to tryptophan caused 5.5- or 46-fold decreases in affinity, respectively (Table 2). The double mutants followed a similar trend as in the USP10 assays with $\mathrm{F} 15 / 33 \mathrm{~W}$ and $\mathrm{F} 33 / 124 \mathrm{~W}$ having a more pronounced loss of affinity as compared to the individual single mutants. F15/124W only modestly reduced the affinity as compared to F15W, further suggesting that F124 does not contribute significantly to Caprin1 peptide binding, at least in the presence of F33. Unexpectedly, the TripleW mutant exhibited slightly increased affinity as compared to $\mathrm{F} 15 / 33 \mathrm{~W}$. This discrepancy may be due to relatively poor solubility of the TripleW in the FP assay. All mutations caused greater relative (fold) decreases in affinity for Caprin1 as compared to USP10.

Our FP assay results revealed that Caprin1 peptide binding was sensitive to mutation of residues F15 and F33. To determine the biological consequences of these mutations, we again overexpressed full length His-tagged G3BP1 mutants in Expi293 cells and assayed for binding efficiency using the affinity capture co-purification (Figure 3B). With the exception of F124W, all mutants displayed a substantial deficiency in Caprin1 co-purification, with reductions of $\sim 98 \%$ as compared to WT (Figure $3 C$ ). Thus, in contrast to USP10, small fold changes in $K_{D}$ values caused dramatic changes in full length G3BP1:Caprin1 complex stability, such that NTF2 domains with the same 
experimental 1:1 affinity for Caprin1 and USP10 systematically co-purified less Caprin 1 (Figure 4).

Sanders et al. recently found a missense mutation in NTF2 (S38F), which blocks stress granule (SG) formation and is unable to form high-affinity complexes with Caprin1 (7). Unfortunately, NTF2 S38F expressed into insoluble inclusion bodies in E. coli. Mutations to Ala, Gly, or Thr were well tolerated, and we tested these mutants in USP10 and Caprin1 FP assays. As expected, all three mutants decreased the affinity for Caprin1, and at similar levels to F15W (Table 3). However, in the USP10 FP assay, NTF2 S38A or S38G increased affinity for USP10 by 2.9 - or 2.4 -fold, respectively, whereas S38T decreased affinity by 2.4 -fold (Table 3 ). S38 is located on a loop away from the NTF2 binding groove, so these results suggest additional G3BP1 residues contribute to peptide binding. Additionally, the data further confirm prevailing hypotheses that small changes in affinities can have dramatic changes on protein-protein interactions.

\section{TripleW can cause significant conformational changes}

Several crystal structures have been published of G3BP1 NTF2 in its apo form and complexed with peptides; however, no mutant crystal structures have been published. Given the dramatic reduction in complex formation as seen with the F33W and TripleW G3BP1 mutants, we wanted to determine the stereochemical cause of the defect. A crystal structure of G3BP1 NTF2 (1-139) TripleW was determined to a resolution of 2.36 $\AA$. The structure was solved by molecular replacement using a deposited structure of G3BP1 NTF2 (PDB ID 4FCJ). The crystal structure is in space group $P 2{ }_{1}{ }_{1} 2_{1}$, with two 
molecules per asymmetric unit. The structure was refined to final $R_{\text {work }}$ and $R_{\text {free }}$ values of $22.2 \%$ and $27.1 \%$, respectively, with excellent model geometry (Table 4 ).

The structure of G3BP1 NTF2 TripleW could be modeled from residues 1-138 except for loop residues 48-50 and 117-123 in Chain B. Density for these loops is absent in other published apo crystal structures of G3BP1 NTF2 from the same space group. NTF2 TripleW dimerizes similarly to WT and has no major changes in backbone conformations, with an all-atom calculated root mean square deviation (rmsd) for the dimer of $0.57 \AA$ (Figure 4).

The TripleW structure reveals major side-chain conformational changes in the binding groove. Residue 33 is dramatically twisted and shifted out of the groove when mutated to a tryptophan in Chain A (Figures 5A and 5C). In Chain B, W33 is similarly shifted out but with a smaller twist (Figures 5B and 5D). While the conformational change in W33 differs in Chain A and Chain B, both conformations are distinct from the WT F33 conformation (Supplementary Figure 4). Mutation of F33 to tryptophan generates more free energy and allows for create flexibility in the W33 side chain. The TripleW crystal structure demonstrates that W33 can adopt at least two low-energy conformations that are distinct from the WT F33 conformation. In Chain A, the large conformational change in F33W causes it to occupy the steric volume that would normally be occupied by F13 from the USP10 FGDE motif peptide (Figure 6A). F33W creates a similar residue-level clash with 1373 from the Caprin1 FlQDSMLD target sequence (Figure 6D). In Chain B, the conformational change in W33 does not create a steric clash with either the USP10 or Caprin1 peptide but W33 occupies a larger volume than the native phenylalanine (Supplementary Figure 5). Whichever of these conformation(s) may be found in solution, 
the binding data confirm that accommodation of the W33 side chain imposes a freeenergy cost on the binding of the peptides.

Mutations of F15W or F124W do not significantly alter the respective side-chain conformations, such that the mutant side chains overlay with the native side chains with steric differences confined to the difference in the size of the ring systems: replacement of phenylalanine with a tryptophan does increase the side chain volume, which in turn shrinks the peptide-binding groove (Figures 5C and 5D). While F15W and F124W mutations cause changes in G3BP1 NTF2, they are much less dramatic than the changes associated with F33W. These differences likely explain why F33W has the most detrimental effect on binding affinity and complex formation.

\section{Mutations in USP10 FGDF motif affect G3BP1 binding}

Having identified key residues in the G3BP1 NTF2 binding groove responsible for binding USP10 and Caprin1 peptides, we turned to investigating residues in USP10 and Caprin 1 that influence G3BP1 binding. While a previous group published an alanine scan in a FGDF-containing nsP3 peptide (32), there has yet to be a full amino-acid substitution analysis of the proposed FGDF or FIQDSMLD core motifs. We designed a peptide array in which the residues in the core motifs are individually substituted with all 20 genetically encoded amino acids. In the USP10 microarray (Figure 7A), we chose to extend our analysis beyond the core motif to include three additional residues on the $\mathrm{N}$ - and $\mathrm{C}$ terminal sides of the motif, so we scanned through the residues of a QYIFGDFSPD reference peptide. We performed the peptide array experiment at a protein concentration of $500 \mathrm{nM}(\sim 9.1 \mu \mathrm{g} / \mathrm{mL})$ of NTF2 protein; visualization of the amount of bound protein by 
antibody staining yielded variable spot intensities (Figure 7A). As expected, the core FGDF motif contributes most of the binding specificity: most substitutions in these four amino acids reduced or abrogated G3BP1 NTF2 binding. The glycine residue (G11) is the most stringent requirement of the core motif, since all substitutions, exception for alanine, dramatically reduce binding. The first phenylalanine residue $(F 10)$ tolerates tyrosine substitution, and to a much lesser extent, valine, leucine, and isoleucine. The second phenylalanine $(\mathrm{F} 13)$ tolerates substitution to tyrosine, valine, leucine, and isoleucine better than F10, and can accommodate a tryptophan. The aspartic acid (D12) is the least strict of the core motif residues, because it can be mutated to most amino acids without dramatic changes in binding ability. The three upstream and downstream residues appear to have only modest and variable effects on G3BP1 NTF2 binding. Most mutations in these residues do not have dramatic changes in binding ability, although there may be some opportunities to enhance affinity by concerted tuning of these peripheral residues $(47,48)$.

\section{Caprin1 has multiple residue interactions with G3BP1}

In the Caprin1 peptide array, we extended our amino-acid scan to include two additional residues on the $\mathrm{N}$ - and $\mathrm{C}$-terminal side of the previously reported core motif yielding YNFIQDSMLDFE as the reference sequence (28). Since our Caprin1 reporter peptide has a higher baseline affinity for G3BP1 NTF2 as calculated by FP, we incubated this array with $300 \mathrm{nM}(\sim 5.5 \mu \mathrm{g} / \mathrm{mL})$ of recombinant G3BP1 NTF2 (Figure 7B). The phenylalanine residue at the $\mathrm{N}$-terminus of the core motif (F372) appears to be the most stringent residue in the core motif, since all substitutions completely abrogated binding to 
G3BP1 NTF2 (Figure 7B). Substitutions at other positions were also disruptive, as expected. Surprisingly, we also found substitutions in the core motif that resulted in higher affinity peptides. For example, both I373F and S376C exhibited dramatic increases in the amount of bound G3BP1 NTF2. Additionally, replacements of M377 with phenylalanine or tyrosine each increased binding. The conserved core motif reported by Solomon et al. had flexibility in three residues: $F(M / I / L) Q(D / E) S z(I / L) D(28)$. I373 substitution by leucine modestly increased affinity, where a methionine at this position ablated binding. Substitutions at the other two residues D375E and L378I modestly reduced binding. Surprisingly, Y370, which lies outside of the earlier core motif, appears to play an important role in binding: at this position all amino acids except for phenylalanine dramatically reduce binding. This suggests that the Caprin1 motif should be extended to include the residue Y370. Consistent with this proposal, mutation of N371 to alanine, glycine, or proline completely ablated binding, suggesting that it also lies within the binding motif. However, all other substitutions were well tolerated, and some substitutions (lle, Leu, Phe, and Trp) created higher affinity peptides. Substitutions of C-terminal flanking residues F380 and E381 were well tolerated and often produced higher affinity spots. Consistent with these results, and in contrast to USP10, trimming of these motif flanking residues also created lower affinity peptides (Supplementary Figure 6). These data suggest that G3BP1 NTF2 interacts with more residues when binding Caprin1 than when binding USP10, which may account for the differential affinity we observed.

Additionally, we analyzed another reported G3BP1 interaction partner, USP13, which like USP10 has also been implicated in the post-maturational stability of CFTR (49, 50). The USP13 binding motif has not been previously reported, so we performed a scan 
of the $\mathrm{N}$-terminal disordered region of the protein using overlapping $22 \mathrm{mer}$ peptides. Multiple spots contained peptides that appeared to interact with G3BP1 NTF2 (Supplementary Figure 7), including the N-terminus, as well as a cluster involving peptides spanning amino acids 73-118. These data suggest that USP13 might have a relatively long motif peptide similar to Caprin1, or alternatively, that multiple motifs might be present within USP13. Further investigations will be needed to definitively identify the USP13 binding motif(s).

\section{Discussion}

In this work, we performed a thorough mutational analysis of the generative interactions that form G3BP1:USP10 and G3BP1:Caprin1 complexes. Using the smallest necessary components for these protein-protein interactions, the G3BP1 NTF2 domain and a motif peptide, we determined the binding affinities for multiple mutants ranging across a spectrum of two orders of magnitude. These results show that the G3BP1 NTF2like domain has a modestly stronger affinity for Caprin1 peptide versus USP10 peptide. Conversely, we showed that binding of the longer Caprin1 peptide is more sensitive than USP10 to mutations in the G3BP1 binding groove. Mutation of G3BP1 residues F15, F33, and/or F124 caused higher fold decreases in binding affinity for Caprin1 versus USP10. Furthermore, larger amounts of USP10 co-purify with overexpressed G3BP1 NTF2 mutants with a given affinity for USP10 compared to the amounts of Caprin1 that co-purify with G3BP1 NTF2 mutants that have similar 1:1 affinity for Caprin1 (Figure 4). In agreement with previous literature, substitution of F33 for a tryptophan in the G3BP1 NTF2-like domain substantially reduces binding for both USP10 and Caprin1. However, 
G3BP1 F33W was able to co-purify a small amount of USP10 ( 10\% compared to WT) but not Caprin1, further supporting the hypothesis that Caprin1 is more sensitive to disruption by mutagenesis than USP10, despite its higher baseline affinity. In any case, relatively modest differences in protein:peptide interaction affinities have been associated with significant differences in the assembly of protein complexes. For example, the Ki-67 and RepoMan phosphatase regulatory proteins exhibit strong selectivity for protein phosphatase 1 (PP1) $\gamma$ isoform over the $\alpha$ isoform, based on a roughly four- to six-fold difference in $K_{D}$ arising primarily from a single amino-acid difference (51).

Co-purification has classically been used in non-affinity chromatography schemes to purify tagless proteins from cells $(52,53)$. Proteins co-purified using these schemes do not necessarily interact or form complexes directly, so additional experiments were necessary to prove protein-protein interactions. In our case, the direct interaction has already been established biochemically and crystallographically using purified components. Recent reports, however, have shown the benefits of affinity chromatography in co-purifying protein complexes such as antibody stabilized proteins, RNA-protein complexes, and cytochrome supercomplex (54-56). The homogeneity of these purified complexes has been confirmed by biophysical methods such as smallangle X-ray scattering (SAXS) and protein crystallography $(8,54)$. G3BP1, USP10, and Caprin1 form protein complexes that are present within SGs that participate in liquid-liquid phase separation (LLPS) (7-9). Several reports have detailed the fluidity and reversibility of SGs (7-9); however, the G3BP1 complexes persisted through a strenuous purification scheme. This suggests that while the larger SG bodies may be fluid, the protein complexes within them may be unexpectedly persistent. Purification of protein complexes 
via metal affinity allows us to study them in vitro without the need to reconstitute the complex using recombinant versions of each individual component. And while not reported here, these purified protein complexes have been useful in development of additional assays. Given the complexity of our system, it was beneficial to use affinity copurification rather than the more common co-immunoprecipitation. While we report our co-purification data as in terms of percentage of WT recovery, we are effectively measuring the off-rate of these protein-protein interactions, which appears to be very slow, consistent with multivalency. The data reported here provide additional evidence that affinity co-purification is a useful tool for in vitro investigations of large proteins complexes.

Based on prior data, we were somewhat surprised by the results of the FP assay and co-purification experiments using the F15W mutant. In the FP assays, G3BP1 NTF2 F15W had calculated $K_{\mathrm{D}}$ values of $11.6 \mu \mathrm{M}$ and $9.3 \mu \mathrm{M}$ for USP10 and Caprin1, respectively. These values are similar and are each less than one order of magnitude different from WT, so we expected reduced, but not abolished, USP10 and Caprin1 copurification. Indeed, the longer Caprin1 binding motif (FIQDSMLD) might have been expected to be more stable to disruption by a single amino-acid substitution. However, the opposite result was observed. While USP10 co-purification was reduced by $\sim 50 \%$ compared to WT, the G3BP1 F15W mutation almost entirely abolished Caprin1 copurification.

One possible explanation is that the off-rate of the larger Caprin1 binding peptide may be faster, even if the affinity is similar. Previous crystal structures have demonstrated the importance of hydrophobic interactions and $\pi$-stacking in the G3BP1 groove for 
USP10 and Caprin1 peptide binding $(17,26,30)$. As a result, this disjunction between USP10 and Caprin1 binding may well reflect differences in the motif peptides, since the USP10 FGDF motif contains two phenylalanines, whereas the Caprin1 FIQDSMLD motif contains only one. G3BP1 F15 participates in parallel-displaced $\pi$-stacking with USP10 F10 and Caprin1 F372. When mutated to a tryptophan, residue 33 occupies a larger hydrophobic volume with a slightly adjusted side chain (Figure 5). This change sterically occludes the positioning the USP10 and Caprin1 peptides (Figure 6), most likely accounting for the observed reduction in affinity. The impact on the USP10 off-rate may be less dramatic, since it has a second phenylalanine, $\mathrm{F} 13$, that can still form $\pi$-stacking interactions with G3BP1 F33. During binding, USP10 F10 and Caprin1 I373 occupy the same position in the G3BP1 binding groove. Interestingly, in the Caprin1 microarray experiment, I373F created a higher affinity peptide. Thus is it possible that Caprin 1 I373F could rescue Caprin1 co-purification in the G3BP1 F15W overexpression system.

The literature describing G3BP1:USP10 and G3BP1:Caprin1 complexes has primarily focused on mutational and functional analyses of G3BP1 residues and binding interfaces $(7-9,29,30)$. We also wanted to understand the contributions from USP10 and Caprin1 in complex formation. Using a systematic amino-acid scan, we have confirmed that the USP10 FGDF motif is the smallest necessary component for G3BP1 NTF2 binding (32). As expected, most substitutions in the core FGDF motif created lower affinity peptides, while substitutions in the upstream and downstream residues modulated affinity, but were mostly well tolerated (Figure 7A). In particular, substitution of flanking residues Y8 and 19 has the potential to create higher affinity peptides. In the Caprin1 array, we saw that most substitutions in the FIQDSMLD motif created lower affinity 
peptides (Figure 7B), again confirming the importance of the previously reported motif (28). However, substitutions I373F, S376C, M377F, and M377Y created higher affinity peptides. Given that 1373 sits similarly to USP10 F13, it is unsurprisingly that replacement with a phenylalanine creates a higher affinity peptide. Similar to the USP10 peptide array, upstream and downstream amino-acid substitutions often created higher affinity peptides; however, flanking residues have a greater role in Caprin1 binding (Supplementary Figure 6). In particular, Y370 appears to play a critical role in G3BP1 binding since it only tolerated substitution to phenylalanine (Figure 7B), and binding was abolished when it was removed (Supplementary Figure 6).

G3BP1:USP10 and G3BP1:Caprin1 complex formation are associated with several disease (57-60). Our peptide array studies suggest that multiple substitutions in the USP10 and Caprin1 binding motifs can create higher affinity peptides. Combining several of these substitutions in one peptide should have additive effects to create significantly higher affinity competitor peptides. Interestingly, the interactions of G3BP1, USP10, and Caprin1 can be cooperative or competitive within the context of multivalent SGs (7-9). Mutations in the G3BP1 NTF2 binding site had disparate effects on Caprin1 and USP10, and their motifs are significantly different in length and composition. As a result, peptide inhibitors based on these different motifs may have differential effects on the composition and stability of the resulting complexes in cells, which can be readily assessed using the co-purification assay described here.

Depending on which patterns are observed, it may ultimately prove therapeutically useful to target one of both component interactions selectively. Given that the necessary component for complex formation is NTF2 and short motif peptides, we could easily adapt 
our FP assay to perform high-throughput screens for novel inhibitors. Indeed, we have previously shown in a separate protein-peptide interaction that our medium-throughput FP assay is easily translated to high-throughput screening studies (61). Currently, there are two published G3BP1 targeted peptide therapies including GAP161 (22), which blocks RasGAP association, and GAP159 (62), which inhibits G3BP1 expression. Resveratrol, an anticancer agent, has been shown to bind the G3BP1 NTF2-like domain and induce apoptosis via p53 activation $(31,63)$. While these therapies are targeted towards cancer progression and metastasis, they do support the concept of targeting G3BP1 and its complex formation as advantageous and novel drug targets.

Benefits arising from the ability to inhibit or control G3BP1 complex formation are observed in nature as well. During viral attack, infected cells will activate stress granule formation to block mRNA processing and halt viral growth (64). As an essential component of stress granules, G3BP1 plays an important role in the antiviral response. Viruses use the FGDF-containing nsP3 to bind G3BP1 and sequester it away from stress granules, thereby inhibiting stress granule formation and allowing viral growth (17). G3BP1 NTF2-like domain has been reported to interact with the nsP3 protein from many viruses including SARS-CoV-2, Old World alphavirus, Semliki Forest virus (SFV), Sindbis virus (SINV), Herpes Simplex virus, and chikungunya (24, 32, 65-69).

Finally, USP10 is a deubiquitinase that plays an important role in protein homeostasis and recycling. One target of USP10 is CFTR, a chloride transporter that helps maintain ion balance across epithelial cells in the lungs and other tissues (36). As part of its normal lifecycle, CFTR is retrieved from the plasma membrane into endosomes for peripheral quality control (70). USP10 deubiquitinates CFTR in endosomes, thereby 
increasing the probability of endocytic recycling, which in turn helps to maintain the abundance of CFTR at the plasma membrane. In lung infections caused by Pseudomonas aeruginosa, a virulence factor called Cif is secreted from the bacteria in outer membrane vesicles, and is able to trigger a reduction in plasma membrane CFTR levels $(20,71)$. Cif stabilizes a G3BP1:USP10 complex in lung cells which renders USP10 inactive and thus unable to deubiquitinate $\operatorname{CFTR}(20,36,71)$. Knockdown of G3BP1 blocks the Cif effect suggesting that USP10 expression and activity is protective for CFTR and ion homeostasis during Pseudomonas aeruginosa infections (20). Thus, while it is not known how Cif drives G3BP1:USP10 complex formation, inhibitors of the interaction could also help to neutralize Cif-facilitated CFTR degradation.

The data reported here will facilitate future experiments on the roles of G3BP1, USP10, and Caprin1. We have identified several mutations in the three proteins that alter binding affinity by large and small amounts. As we have shown, small changes in affinity can have dramatic effects on the cell biology of the system, so we can use these mutations to selectively manipulate the G3BP1-USP10-Caprin1 system. This will help deconvolute the intricate interaction network surrounding these three proteins and help address unanswered questions in disease contexts including bacterial virulence, antiviral response, innate immunity, and neurodegeneration.

\section{Materials and Methods}

\section{Cloning, protein expression, and purification}

The pNIC28-G3BP1-NTF2 vector was graciously provided by Dr. Gerald Mclnerney (Karolinska Institutet, Stockholm, Sweden). Mutants were created via side directed 
mutagenesis using primers designed on NEBaseChanger and Q5 site-directed mutagenesis kit following manufacturer's directions (New England BioLabs). BL21(DE3) cells were transformed with plasmid and grown on LB (72) $+50 \mu \mathrm{g} / \mathrm{mL}$ Kanamycin agar plates at $37^{\circ} \mathrm{C}$ overnight. Transformants were used to inoculate $10 \mathrm{~mL} \mathrm{LB}+50 \mu \mathrm{g} / \mathrm{mL}$ Kanamycin broth and grown at $37^{\circ} \mathrm{C}$ overnight. Overnight cultures were used to inoculate $1 \mathrm{~L} \mathrm{LB}+50 \mu \mathrm{g} / \mathrm{mL}$ Kanamycin broth in non-baffled $2 \mathrm{~L}$ flasks and allowed to grow at $37^{\circ} \mathrm{C}$ with shaking. Once the $\mathrm{OD}_{600}$ of the cultures reached $\sim 0.8$, the cultures were induced with $0.1 \mathrm{mM}$ IPTG and transferred to $30^{\circ} \mathrm{C}$ with shaking for 4 hours. Cultures with mutant G3BP1 NTF2 were expressed at $16^{\circ} \mathrm{C}$ with shaking overnight ( 18 hours). After expression, cultures were pelleted in a JLA-9.1000 rotor at 4,500 rpm for 15 min at $4^{\circ} \mathrm{C}$. Pellet was resuspended in lysis buffer (500 mM NaCl, 20 mM Tris pH 8.5, 2 mM MgCl ) supplemented with Pierce universal nuclease at 25 units $/ \mathrm{mL}$ and Roche cOmplete EDTAfree Protease Inhibitor Cocktail tablets. Cell lysis was carried out using an M-110L microfluidizer (Microfluidics) in 3 passes at $\sim 18 \mathrm{kpsi}$. Lysate was spun down for 1 hour at $40 \mathrm{~K}$ rpm at $4^{\circ} \mathrm{C}$ in a Type 45 Ti rotor. $5 \mathrm{~mL}$ of HisPur Ni-NTA Resin (Thermo Scientific) was washed with $25 \mathrm{~mL}$ wash buffer (500 mM NaCl, $20 \mathrm{mM}$ Tris, $\mathrm{pH}$ 8.5). The clarified cell lysate was supplemented with $20 \mathrm{mM}$ imidazole, $\mathrm{pH} 8.5$, and then incubated with $\mathrm{Ni}$ NTA resin with gentle stirring for 1 hour at $4^{\circ} \mathrm{C}$. The mixture was returned to room temperature and passed through a gravity flow column. The protein-bound resin was washed four times with $25 \mathrm{~mL}$ of wash buffer supplemented with $80 \mathrm{mM}$ imidazole, $\mathrm{pH}$ 8.5. The protein was eluted with $500 \mathrm{mM} \mathrm{NaCl}, 20 \mathrm{mM}$ Tris $\mathrm{pH} 8.5$, and $500 \mathrm{mM}$ imidazole, $\mathrm{pH}$ 8.5. Eluates were immediately diluted 1:1 with $800 \mathrm{mM} \mathrm{NaCl}$ and $20 \mathrm{mM}$ Tris, $\mathrm{pH} 8.5$ then pooled for dialysis at room temperature into $500 \mathrm{mM} \mathrm{NaCl}$ and $20 \mathrm{mM}$ 
Tris, pH 8.5. After at least an hour of dialysis, purified recombinant TEV was added at a ratio of $1: 20$ and left at room temperature overnight. The cleaved protein was passed through HisPur Ni-NTA resin equilibrated with wash buffer. The flow through was collected, concentrated, and loaded on a HiLoad 26/600 Superdex 200 pg size-exclusion chromatography column (GE Healthcare). Protein was eluted using a buffer of $150 \mathrm{mM}$ $\mathrm{NaCl}$ and $20 \mathrm{mM}$ Tris pH 8.5 at the expected molecular mass (Supplementary Figure 8).

Full-length G3BP1 was expressed and purified from Expi293F cells (Life Tech) as detailed here. The pCMV-His-G3BP1 vector was purchased from SinoBiolgical. Mutants were generated using the same site-directed mutagenesis protocol as detailed above. Expi293F cells were grown in Expi293 Expression Media at $37^{\circ} \mathrm{C}, 8 \% \mathrm{CO}_{2}$ with shaking at $125 \mathrm{rpm}$ in a disposable plastic Erlenmeyer flask (non-baffled). On the day of transfection, cell density was between $4-5 \times 10^{6} \mathrm{cells} / \mathrm{mL}$ with $>95 \%$ viability as determined using a TC20 automated cell counter (Bio-Rad). Cells were diluted with warm Expi293 Expression Media to $2 \times 10^{6}$ cells $/ \mathrm{mL}$ in $42.5 \mathrm{~mL}$. $5 \mu \mathrm{g}$ of plasmid was added to $2.5 \mathrm{~mL}$ Opti-MEM Reduced-Serum Medium (Gibco). $15 \mathrm{mg}$ of sterile PEI was added to $2.5 \mathrm{~mL}$ Opti-MEM Reduced-Serum Medium. PEI solution was added to DNA solution, inverted several times, and incubated for 20 minutes at room temperature. DNA:PEI solution added to flask of Expi293 cells then returned to incubator at $37^{\circ} \mathrm{C}, 8 \% \mathrm{CO}_{2}$ with shaking at $125 \mathrm{rpm}$. After $16-20$ hours, $25 \mu \mathrm{L}$ of $100 \mathrm{mM}$ sterile-filtered valproic acid (Sigma) and $2.5 \mathrm{~mL}$ of $100 \mathrm{mM}$ sterile-filtered sodium propionate (Sigma) in Expi293TM Expression Medium were added to the flask. 72 hours after transfection, cells were gently pelleted, and then resuspended in lysis buffer $(500 \mathrm{mM} \mathrm{NaCl}, 20 \mathrm{mM}$ Tris $\mathrm{pH} 8.5,1 \%$ 
$[\mathrm{v} / \mathrm{v}]$ IGEPAL, $2 \mathrm{mM} \mathrm{MgCl}$ ) supplemented with Pierce universal nuclease at 25 units $/ \mathrm{mL}$ and Roche cOmplete EDTA-free Protease Inhibitor Cocktail tablets. Conical tubes were placed on gentle rotator for $30 \mathrm{~min}$ at $4^{\circ} \mathrm{C}$. Clarification and elution followed the same protocol as above. After elution, samples from first eluate were collected, boiled in SDS sample buffer, and run on a $10 \%$ SDS acrylamide gel. Proteins were transferred to a PVDF membrane at $95 \mathrm{~V}$ for 2 hours at $4^{\circ} \mathrm{C}$. Membranes were blocked for 1 hour at room temperature in Odyssey Blocking Buffer (LiCor). Primary antibodies (rabbit polyclonal USP10 antibody, Bethyl; rabbit polyclonal Caprin1 antibody, Proteintech; mouse monoclonal G3BP1 antibody, Santa Cruz) were diluted into Odyssey Blocking Buffer with $0.2 \%(v / v)$ Tween 20 . Membranes were incubated with primary antibody solutions overnight at $4^{\circ} \mathrm{C}$. Membranes were washed with Tris-buffered saline, $0.1 \%(v / v)$ Tween 20 (TBST) then incubated for 1 hour in IRDye secondary antibody (LiCor) in Odyssey Blocking Buffer with 0.2\% ( $v / v)$ Tween $20+0.01 \%(w / v)$ SDS. Membranes were washed with TBST multiple times, and then imaged with the Odyssey CLx Imager (LiCor).

\section{Fluorescence polarization}

Fluorescence polarization assays were performed following lab established protocols (73). Briefly, experiments were performed using G3BP1 NTF2 protein and performed in triplicate. Biomatik synthesized the reporter peptides used for all FP experiments: fluorescein-aminohexanoic acid $\left(F^{*}\right)$-YIFGDFSP (USP10) and $F^{*}$-YNFIQDSMLDFE (Caprin1). All experiments were performed in stock buffer (150 mM NaCl, $20 \mathrm{mM}$ Tris, $\mathrm{pH}$ 8.5) supplemented with $30 \mu \mathrm{M}$ thesit and $0.1 \mathrm{mg} / \mathrm{mL} \operatorname{lgG}$. Protein was serially diluted into stock buffer containing $30 \mathrm{nM}$ reporter peptide then transferred to a 384-well plate. 
Protein-reporter was allowed to incubate at room temperature for $30 \mathrm{~min}$. Plates were scanned on a Synergy Neo2 multi-mode plate reader (BioTek) and the anisotropy was measured. A non-linear least-squares algorithm was used to fit the merged anisotropy data and calculate $K_{D}$. Anisotropy and intensity values were analyzed manually to confirm no effect due to light scattering.

\section{Crystallography}

G3BP1 NTF2 TripleW crystals were obtained by vapor diffusion in a sitting drop composed of $200 \mathrm{~nL}$ of $1.6 \mathrm{mg} / \mathrm{mL}$ protein in $100 \mathrm{mM} \mathrm{NaCl}, 20 \mathrm{mM}$ Tris, $\mathrm{pH} 8.5$ and 200 $\mathrm{nL}$ of well solution (20\% [W/V] PEG $8000,100 \mathrm{mM}$ HEPES $\mathrm{pH} 7.5)$ and equilibrated by vapor diffusion with $50 \mu \mathrm{L}$ of well solution. The drop was set up using a NT8 drop setter (Formulatrix) and the well solution was from the Wizard Classic I\&II High-Throughput Screen (Molecular Dimensions). The tray was incubated and imaged using Rock Imager and Rock Maker instrumentation (Formulatrix). The crystal was harvested and immediately flash cooled in liquid nitrogen. The oscillation data collection was performed at the National Synchrotron Light Source II beamline 17-ID-2 (FMX) equipped with an Eiger $16 \mathrm{M}$ detector at $100 \mathrm{~K}$, an oscillation range of $0.2^{\circ}$ per frame, a total of wedge of $180^{\circ}$. The diffraction images were processed using XDS (74). The Rfree set was generated from $5 \%$ of the reflections in thin resolution shells using the Phenix (75) reflection file editor. Initial phases were generated by Phaser (76) via molecular replacement using G3BP1 NTF2 WT (PDB ID 4FCJ) as a search model. Iterative automatic and manual refinement were performed using Phenix and Coot $(75,77)$. 


\section{Peptide Array}

The 600 peptide SPOT cellulose array was generated by the Biopolymers \& Proteomics Core Facility at the Koch Institute for Integrative Cancer Research of Massachusetts Institute of Technology. An Intavis SPOT synthesis peptide arrayer system was used to make the array.

The peptide array was hydrated with methanol for $10 \mathrm{~min}$, then washed three times with TBST, and then blocked for 2 hours in Odyssey Blocking Buffer. The array was incubated with His-tagged G3BP1 NTF2 in blocking buffer with 0.2\% $(v / v)$ Tween 20 for 20 hours at $4^{\circ} \mathrm{C}$, and then washed three times with TBST. G3BP1 was detected using a His-tag antibody (Santa Cruz) followed by IRDye secondary antibody in Odyssey Blocking Buffer with $0.2 \%(v / v)$ Tween $20+0.01 \%(w / v)$ SDS. Arrays were scanned on an Odyssey CLx Imager.

\section{Accession Numbers}

The coordinates and structure factors for the G3BP1 NTF2-like domain TripleW structure are available at the Protein Data Bank under PDB ID: 7S17.

\section{Funding}

This work was supported by National Institutes of Health grants R01-Al091699, P20GM113132, and P30-DK117469, training grant T32-HL134598, and beamline support P30-GM133893. Additional beamline funding was provided by US Department of Energy contracts DE-SC0012704 and KP1607011. The content is solely the responsibility of the 
authors and does not necessarily represent the official views of the National Institutes of Health or the US Department of Energy.

\section{Acknowledgments}

We would like to thank Drs. Babak Andi, Martin Fuchs, and Vivian Stojanoff at FMX for support of diffraction experiments, Dr. Noor Taher for advice and suggestions regarding data processing and structure refinement, Dr. Kelli Hvorecny for mentorship and advice, Dr. Andreia Verissimo and Dr. Angela Kull for the support of the bioMT core facilities, Dr. Bradley Bartholomai and members of the Madden and Stanton lab for helpful discussions. 


\section{References}

1. Dunker, A. K., Cortese, M. S., Romero, P., Iakoucheva, L. M., and Uversky, V. N. (2005) Flexible nets. The roles of intrinsic disorder in protein interaction networks. FEBS J. 272, 5129-5148

2. Kim, P. M., Sboner, A., Xia, Y., and Gerstein, M. (2008) The role of disorder in interaction networks: a structural analysis. Mol. Syst. Biol. 4, 179

3. Wright, P. E., and Dyson, H. J. (2015) Intrinsically Disordered Proteins in Cellular Signaling and Regulation. Nat. Rev. Mol. Cell Biol. 16, 18-29

4. Wright, P. E., and Dyson, H. J. (1999) Intrinsically unstructured proteins: re-assessing the protein structure-function paradigm. J. Mol. Biol. 293, 321-331

5. Dyson, H. J., and Wright, P. E. (2005) Intrinsically unstructured proteins and their functions. Nat. Rev. Mol. Cell Biol. 6, 197-208

6. Oldfield, C. J., Cheng, Y., Cortese, M. S., Romero, P., Uversky, V. N., and Dunker, A. K. (2005) Coupled folding and binding with alpha-helix-forming molecular recognition elements. Biochemistry. 44, 12454-12470

7. Sanders, D. W., Kedersha, N., Lee, D. S. W., Strom, A. R., Drake, V., Riback, J. A., Bracha, D., Eeftens, J. M., Iwanicki, A., Wang, A., Wei, M.-T., Whitney, G., Lyons, S. M., Anderson, P., Jacobs, W. M., Ivanov, P., and Brangwynne, C. P. (2020) Competing ProteinRNA Interaction Networks Control Multiphase Intracellular Organization. Cell. 181, 306324.e28

8. Yang, P., Mathieu, C., Kolaitis, R.-M., Zhang, P., Messing, J., Yurtsever, U., Yang, Z., Wu, J., Li, Y., Pan, Q., Yu, J., Martin, E. W., Mittag, T., Kim, H. J., and Taylor, J. P. (2020) G3BP1 Is a Tunable Switch that Triggers Phase Separation to Assemble Stress Granules. Cell. 181, 325-345.e28

9. Guillén-Boixet, J., Kopach, A., Holehouse, A. S., Wittmann, S., Jahnel, M., Schlüßler, R., Kim, K., Trussina, I. R. E. A., Wang, J., Mateju, D., Poser, I., Maharana, S., Ruer-Gruß, M., Richter, D., Zhang, X., Chang, Y.-T., Guck, J., Honigmann, A., Mahamid, J., Hyman, A. A., Pappu, R. V., Alberti, S., and Franzmann, T. M. (2020) RNA-Induced Conformational Switching and Clustering of G3BP Drive Stress Granule Assembly by Condensation. Cell. 181, 346-361.e17

10. Parker, F., Maurier, F., Delumeau, I., Duchesne, M., Faucher, D., Debussche, L., Dugue, A., Schweighoffer, F., and Tocque, B. (1996) A Ras-GTPase-activating protein SH3-domainbinding protein. Mol. Cell. Biol. 16, 2561-2569

11. Gallouzi, I. E., Parker, F., Chebli, K., Maurier, F., Labourier, E., Barlat, I., Capony, J. P., Tocque, B., and Tazi, J. (1998) A novel phosphorylation-dependent RNase activity of GAP-SH3 binding protein: a potential link between signal transduction and RNA stability. Mol. Cell. Biol. 18, 3956-3965

12. Tourrière, H., Gallouzi, I. E., Chebli, K., Capony, J. P., Mouaikel, J., van der Geer, P., and Tazi, J. (2001) RasGAP-associated endoribonuclease G3Bp: selective RNA degradation and phosphorylation-dependent localization. Mol. Cell. Biol. 21, 7747-7760

13. Tourrière, H., Chebli, K., Zekri, L., Courselaud, B., Blanchard, J. M., Bertrand, E., and Tazi, J. (2003) The RasGAP-associated endoribonuclease G3BP assembles stress granules. $J$. Cell Biol. 160, 823-831

14. Prigent, M., Barlat, I., Langen, H., and Dargemont, C. (2000) IkappaBalpha and IkappaBalpha /NF-kappa B complexes are retained in the cytoplasm through interaction with a novel partner, RasGAP SH3-binding protein 2. J. Biol. Chem. 275, 36441-36449 
15. Soncini, C., Berdo, I., and Draetta, G. (2001) Ras-GAP SH3 domain binding protein (G3BP) is a modulator of USP10, a novel human ubiquitin specific protease. Oncogene. 20, 38693879

16. Zekri, L., Chebli, K., Tourrière, H., Nielsen, F. C., Hansen, T. V. O., Rami, A., and Tazi, J. (2005) Control of fetal growth and neonatal survival by the RasGAP-associated endoribonuclease G3BP. Mol. Cell. Biol. 25, 8703-8716

17. Schulte, T., Liu, L., Panas, M. D., Thaa, B., Dickson, N., Götte, B., Achour, A., and McInerney, G. M. (2016) Combined structural, biochemical and cellular evidence demonstrates that both FGDF motifs in alphavirus nsP3 are required for efficient replication. Open Biol. 6, 160078

18. Irvine, K., Stirling, R., Hume, D., and Kennedy, D. (2004) Rasputin, more promiscuous than ever: a review of G3BP. Int. J. Dev. Biol. 48, 1065-1077

19. Piatnitskaia, S., Takahashi, M., Kitaura, H., Katsuragi, Y., Kakihana, T., Zhang, L., Kakita, A., Iwakura, Y., Nawa, H., Miura, T., Ikeuchi, T., Hara, T., and Fujii, M. (2019) USP10 is a critical factor for Tau-positive stress granule formation in neuronal cells. Sci. Rep. 9, 10591

20. Bomberger, J. M., Ye, S., MacEachran, D. P., Koeppen, K., Barnaby, R. L., O’Toole, G. A., and Stanton, B. A. (2011) A Pseudomonas aeruginosa Toxin that Hijacks the Host Ubiquitin Proteolytic System. PLOS Pathog. 7, e1001325

21. Li, Y., Wang, J., Zhong, S., Li, J., and Du, W. (2020) Overexpression of G3BP1 facilitates the progression of colon cancer by activating $\beta$-catenin signaling. Mol. Med. Rep. 22, 44034411

22. Zhang, H., Zhang, S., He, H., Zhao, W., Chen, J., and Shao, R. (2012) GAP161 targets and downregulates G3BP to suppress cell growth and potentiate cisplaitin-mediated cytotoxicity to colon carcinoma HCT116 cells. Cancer Sci. 103, 1848-1856

23. Zhang, H., Zhang, S. -h, He, H. -w, Zhang, C. -x, Yu, D. -k, and Shao, R. -g (2013) Downregulation of G3BPs inhibits the growth, migration and invasion of human lung carcinoma H1299 cells by suppressing the Src/FAK-associated signaling pathway. Cancer Gene Ther. 20, 622-629

24. Alam, U., and Kennedy, D. (2019) Rasputin a decade on and more promiscuous than ever? A review of G3BPs. Biochim. Biophys. Acta Mol. Cell Res. 1866, 360-370

25. Kennedy, D., French, J., Guitard, E., Ru, K., Tocque, B., and Mattick, J. (2001) Characterization of G3BPs: tissue specific expression, chromosomal localisation and rasGAP(120) binding studies. J. Cell. Biochem. 84, 173-187

26. Kristensen, O. (2015) Crystal structure of the G3BP2 NTF2-like domain in complex with a canonical FGDF motif peptide. Biochem. Biophys. Res. Commun. 467, 53-57

27. Vognsen, T., Møller, I. R., and Kristensen, O. (2013) Crystal structures of the human G3BP1 NTF2-like domain visualize FxFG Nup repeat specificity. PloS One. 8, e80947

28. Solomon, S., Xu, Y., Wang, B., David, M. D., Schubert, P., Kennedy, D., and Schrader, J. W. (2007) Distinct structural features of caprin-1 mediate its interaction with G3BP-1 and its induction of phosphorylation of eukaryotic translation initiation factor 2alpha, entry to cytoplasmic stress granules, and selective interaction with a subset of mRNAs. Mol. Cell. Biol. 27, 2324-2342

29. Kedersha, N., Panas, M. D., Achorn, C. A., Lyons, S., Tisdale, S., Hickman, T., Thomas, M., Lieberman, J., McInerney, G. M., Ivanov, P., and Anderson, P. (2016) G3BP-Caprin1USP10 complexes mediate stress granule condensation and associate with 40S subunits. $J$. Cell Biol. 212, 845-860 
30. Schulte, T., Panas, M. D., Williams, L., Kedersha, N., Fleck, J. S., Tan, T. J. C., Olsson, A., Morro, A. M., Hanke, L., Nilvebrant, J., Giang, K. A., Nygren, P.-Å., Anderson, P., Achour, A., and McInerney, G. M. (2021) Caprin-1 binding to the critical stress granule protein G3BP1 is regulated by $\mathrm{pH}, 10.1101 / 2021.02 .05 .429362$

31. Oi, N., Yuan, J., Malakhova, M., Luo, K., Li, Y., Ryu, J., Zhang, L., Bode, A. M., Xu, Z., Li, Y., Lou, Z., and Dong, Z. (2015) Resveratrol induces apoptosis by directly targeting RasGTPase-activating protein SH3 domain-binding protein 1. Oncogene. 34, 2660-2671

32. Panas, M. D., Schulte, T., Thaa, B., Sandalova, T., Kedersha, N., Achour, A., and McInerney, G. M. (2015) Viral and cellular proteins containing FGDF motifs bind G3BP to block stress granule formation. PLoS Pathog. 11, e1004659

33. Yuan, J., Luo, K., Zhang, L., Cheville, J. C., and Lou, Z. (2010) USP10 regulates p53 localization and stability by deubiquitinating p53. Cell. 140, 384-396

34. Liu, J., Xia, H., Kim, M., Xu, L., Li, Y., Zhang, L., Cai, Y., Norberg, H. V., Zhang, T., Furuya, T., Jin, M., Zhu, Z., Wang, H., Yu, J., Li, Y., Hao, Y., Choi, A., Ke, H., Ma, D., and Yuan, J. (2011) Beclin1 controls the levels of p53 by regulating the deubiquitination activity of USP10 and USP13. Cell. 147, 223-234

35. Boulkroun, S., Ruffieux-Daidié, D., Vitagliano, J.-J., Poirot, O., Charles, R.-P., Lagnaz, D., Firsov, D., Kellenberger, S., and Staub, O. (2008) Vasopressin-inducible ubiquitin-specific protease 10 increases ENaC cell surface expression by deubiquitylating and stabilizing sorting nexin 3. Am. J. Physiol. Renal Physiol. 295, F889-900

36. Bomberger, J. M., Barnaby, R. L., and Stanton, B. A. (2009) The deubiquitinating enzyme USP10 regulates the post-endocytic sorting of cystic fibrosis transmembrane conductance regulator in airway epithelial cells. J. Biol. Chem. 284, 18778-18789

37. Lin, Z., Yang, H., Tan, C., Li, J., Liu, Z., Quan, Q., Kong, S., Ye, J., Gao, B., and Fang, D. (2013) USP10 antagonizes c-Myc transcriptional activation through SIRT6 stabilization to suppress tumor formation. Cell Rep. 5, 1639-1649

38. Grunda, J. M., Nabors, L. B., Palmer, C. A., Chhieng, D. C., Steg, A., Mikkelsen, T., Diasio, R. B., Zhang, K., Allison, D., Grizzle, W. E., Wang, W., Gillespie, G. Y., and Johnson, M.

R. (2006) Increased expression of thymidylate synthetase (TS), ubiquitin specific protease 10 (USP10) and survivin is associated with poor survival in glioblastoma multiforme (GBM). J. Neurooncol. 80, 261-274

39. Weisberg, E. L., Schauer, N. J., Yang, J., Lamberto, I., Doherty, L., Bhatt, S., Nonami, A., Meng, C., Letai, A., Wright, R., Tiv, H., Gokhale, P. C., Ritorto, M. S., De Cesare, V., Trost, M., Christodoulou, A., Christie, A., Weinstock, D. M., Adamia, S., Stone, R., Chauhan, D., Anderson, K. C., Seo, H.-S., Dhe-Paganon, S., Sattler, M., Gray, N. S., Griffin, J. D., and Buhrlage, S. J. (2017) Inhibition of USP10 induces degradation of oncogenic FLT3. Nat. Chem. Biol. 13, 1207-1215

40. Takahashi, M., Kitaura, H., Kakita, A., Kakihana, T., Katsuragi, Y., Nameta, M., Zhang, L., Iwakura, Y., Nawa, H., Higuchi, M., Komatsu, M., and Fujii, M. (2018) USP10 Is a Driver of Ubiquitinated Protein Aggregation and Aggresome Formation to Inhibit Apoptosis. iScience. 9, 433-450

41. Wang, B., David, M. D., and Schrader, J. W. (2005) Absence of caprin-1 results in defects in cellular proliferation. J. Immunol. Baltim. Md 1950. 175, 4274-4282

42. Qiu, Y.-Q., Yang, C.-W., Lee, Y.-Z., Yang, R.-B., Lee, C.-H., Hsu, H.-Y., Chang, C.-C., and Lee, S.-J. (2015) Targeting a ribonucleoprotein complex containing the caprin-1 protein and 
the c-Myc mRNA suppresses tumor growth in mice: an identification of a novel oncotarget. Oncotarget. 6, 2148-2163

43. El Fatimy, R., Tremblay, S., Dury, A. Y., Solomon, S., De Koninck, P., Schrader, J. W., and Khandjian, E. W. (2012) Fragile X mental retardation protein interacts with the RNAbinding protein Caprin1 in neuronal RiboNucleoProtein complexes [corrected]. PloS One. 7, e39338

44. Yang, Z.-S., Qing, H., Gui, H., Luo, J., Dai, L.-J., and Wang, B. (2019) Role of caprin-1 in carcinogenesis. Oncol. Lett. 18, 15-21

45. Bhattacharya, U., Neizer-Ashun, F., Mukherjee, P., and Bhattacharya, R. (2020) When the chains do not break: the role of USP10 in physiology and pathology. Cell Death Dis. 11, 1033

46. Kim, T. H., Tsang, B., Vernon, R. M., Sonenberg, N., Kay, L. E., and Forman-Kay, J. D. (2019) Phospho-dependent phase separation of FMRP and CAPRIN1 recapitulates regulation of translation and deadenylation. Science. 365, 825-829

47. Cushing, P. R., Vouilleme, L., Pellegrini, M., Boisguerin, P., and Madden, D. R. (2010) A Stabilizing Influence: CAL PDZ Inhibition Extends the Half-Life of $\Delta$ F508-CFTR. Angew. Chem. Int. Ed Engl. 49, 9907-9911

48. Roberts, K. E., Cushing, P. R., Boisguerin, P., Madden, D. R., and Donald, B. R. (2012) Computational Design of a PDZ Domain Peptide Inhibitor that Rescues CFTR Activity. PLoS Comput. Biol. 8, e1002477

49. Xie, X., Matsumoto, S., Endo, A., Fukushima, T., Kawahara, H., Saeki, Y., and Komada, M. (2018) Deubiquitylases USP5 and USP13 are recruited to and regulate heat-induced stress granules through their deubiquitylating activities. J. Cell Sci. 10.1242/jcs.210856

50. Pesce, E., Sondo, E., Ferrera, L., Tomati, V., Caci, E., Scudieri, P., Musante, I., Renda, M., Baatallah, N., Servel, N., Hinzpeter, A., di Bernardo, D., Pedemonte, N., and Galietta, L. J. V. (2018) The Autophagy Inhibitor Spautin-1 Antagonizes Rescue of Mutant CFTR Through an Autophagy-Independent and USP13-Mediated Mechanism. Front. Pharmacol. 9, 1464

51. Kumar, G. S., Gokhan, E., De Munter, S., Bollen, M., Vagnarelli, P., Peti, W., and Page, R. (2016) The Ki-67 and RepoMan mitotic phosphatases assemble via an identical, yet novel mechanism. eLife. 5, e16539

52. van den Bos, C., Rammes, A., Vogl, T., Boynton, R., Zaia, J., Sorg, C., and Roth, J. (1998) Copurification of P6, MRP8, and MRP14 from human granulocytes and separation of individual proteins. Protein Expr. Purif. 13, 313-318

53. Clare, D. A., and Lecce, J. G. (1991) Copurification of bovine milk xanthine oxidase and immunoglobulin. Arch. Biochem. Biophys. 286, 233-237

54. de Marco, A. (2021) Recombinant Proteins Co-Expressed and Co-Purified in the Presence of Antibody Fragments. Methods Mol. Biol. Clifton NJ. 2178, 93-103

55. El Khouri, M., Catala, M., Seijo, B., Chabal, J., Dardel, F., Tisné, C., and Ponchon, L. (2021) Coexpression and Copurification of RNA-Protein Complexes in Escherichia coli. Methods Mol. Biol. Clifton NJ. 2323, 67-73

56. Falke, D., Fischer, M., Ihling, C., Hammerschmidt, C., Sinz, A., and Sawers, G. (2021) Copurification of nitrate reductase 1 with components of the cytochrome bcc-aa3 oxidase supercomplex from spores of Streptomyces coelicolor A3(2). FEBS Open Bio. 11, 652-669

57. Markmiller, S., Soltanieh, S., Server, K. L., Mak, R., Jin, W., Fang, M. Y., Luo, E.-C., Krach, F., Yang, D., Sen, A., Fulzele, A., Wozniak, J. M., Gonzalez, D. J., Kankel, M. W., 
Gao, F.-B., Bennett, E. J., Lécuyer, E., and Yeo, G. W. (2018) Context-Dependent and Disease-Specific Diversity in Protein Interactions within Stress Granules. Cell. 172, 590604.e13

58. Protter, D. S. W., and Parker, R. (2016) Principles and Properties of Stress Granules. Trends Cell Biol. 26, 668-679

59. Riggs, C. L., Kedersha, N., Ivanov, P., and Anderson, P. (2020) Mammalian stress granules and $\mathrm{P}$ bodies at a glance. $J$. Cell Sci. 10.1242/jcs.242487

60. Wolozin, B., and Ivanov, P. (2019) Stress granules and neurodegeneration. Nat. Rev. Neurosci. 20, 649-666

61. Zhao, Y., Cushing, P. R., Smithson, D. C., Pellegrini, M., Pletnev, A. A., Al-Ayyoubi, S., Grassetti, A. V., Gerber, S. A., Guy, R. K., and Madden, D. R. (2018) Cysteine modifiers suggest an allosteric inhibitory site on the CAL PDZ domain. Biosci. Rep. 38, BSR20180231

62. Zhang, H., Zhang, S., He, H., Zhang, C., Chen, Y., Yu, D., Chen, J., and Shao, R. (2014) RasGAP-derived peptide GAP159 enhances cisplatin-induced cytotoxicity and apoptosis in HCT116 cells. Acta Pharm. Sin. B. 4, 128-134

63. Amen, T., Guihur, A., Zelent, C., Ursache, R., Wilting, J., and Kaganovich, D. (2021) Resveratrol and related stilbene-derivatives induce Stress Granules with distinct clearance kinetics. Mol. Biol. Cell. 10.1091/mbc.E21-02-0066

64. Eiermann, N., Haneke, K., Sun, Z., Stoecklin, G., and Ruggieri, A. (2020) Dance with the Devil: Stress Granules and Signaling in Antiviral Responses. Viruses. 12, 984

65. Almutairy, B. K., Alshetaili, A., Anwer, K., and Ali, N. (2021) In Silico Identification of MicroRNAs targeting the Key nucleator of Stress Granules, G3BP: Promising Therapeutics for SARS-CoV-2 Infection. Saudi J. Biol. Sci. 10.1016/j.sjbs.2021.08.056

66. Luo, L., Li, Z., Zhao, T., Ju, X., Ma, P., Jin, B., Zhou, Y., He, S., Huang, J., Xu, X., Zou, Y., Li, P., Liang, A., Liu, J., Chi, T., Huang, X., Ding, Q., Jin, Z., Huang, C., and Zhang, Y. (2021) SARS-CoV-2 nucleocapsid protein phase separates with G3BPs to disassemble stress granules and facilitate viral production. Sci. Bull. 66, 1194-1204

67. Panas, M. D., Ahola, T., and McInerney, G. M. (2014) The C-terminal repeat domains of nsP3 from the Old World alphaviruses bind directly to G3BP. J. Virol. 88, 5888-5893

68. Cristea, I. M., Carroll, J.-W. N., Rout, M. P., Rice, C. M., Chait, B. T., and MacDonald, M. R. (2006) Tracking and elucidating alphavirus-host protein interactions. J. Biol. Chem. 281, 30269-30278

69. Fros, J. J., Domeradzka, N. E., Baggen, J., Geertsema, C., Flipse, J., Vlak, J. M., and Pijlman, G. P. (2012) Chikungunya virus nsP3 blocks stress granule assembly by recruitment of G3BP into cytoplasmic foci. J. Virol. 86, 10873-10879

70. Bradbury, N. A., Jilling, T., Berta, G., Sorscher, E. J., Bridges, R. J., and Kirk, K. L. (1992) Regulation of plasma membrane recycling by CFTR. Science. 256, 530-532

71. Bomberger, J. M., MacEachran, D. P., Coutermarsh, B. A., Ye, S., O’Toole, G. A., and Stanton, B. A. (2009) Long-Distance Delivery of Bacterial Virulence Factors by Pseudomonas aeruginosa Outer Membrane Vesicles. PLOS Pathog. 5, e1000382

72. Miller, J. H. (1972) Experiments in molecular genetics, Cold Spring Harbor Laboratory, [Cold Spring Harbor, N.Y.]

73. Amacher, J. F., Cushing, P. R., Brooks, L., Boisguerin, P., and Madden, D. R. (2014) Identifying Stereochemical Preferences that Modulate Affinity and Selectivity among Five 
PDZ Domains that Bind CFTR: Comparative Crystallographic and Sequence Analyses. Struct. Lond. Engl. 1993. 22, 82-93

74. Kabsch, W. (2010) XDS. Acta Crystallogr. D Biol. Crystallogr. 66, 125-132

75. Liebschner, D., Afonine, P. V., Baker, M. L., Bunkóczi, G., Chen, V. B., Croll, T. I., Hintze, B., Hung, L.-W., Jain, S., McCoy, A. J., Moriarty, N. W., Oeffner, R. D., Poon, B. K., Prisant, M. G., Read, R. J., Richardson, J. S., Richardson, D. C., Sammito, M. D., Sobolev, O. V., Stockwell, D. H., Terwilliger, T. C., Urzhumtsev, A. G., Videau, L. L., Williams, C. J., and Adams, P. D. (2019) Macromolecular structure determination using X-rays, neutrons and electrons: recent developments in Phenix. Acta Crystallogr. Sect. Struct. Biol. 75, 861877

76. McCoy, A. J., Grosse-Kunstleve, R. W., Adams, P. D., Winn, M. D., Storoni, L. C., and Read, R. J. (2007) Phaser crystallographic software. J. Appl. Crystallogr. 40, 658-674

77. Emsley, P., Lohkamp, B., Scott, W. G., and Cowtan, K. (2010) Features and development of Coot. Acta Crystallogr. D Biol. Crystallogr. 66, 486-501 

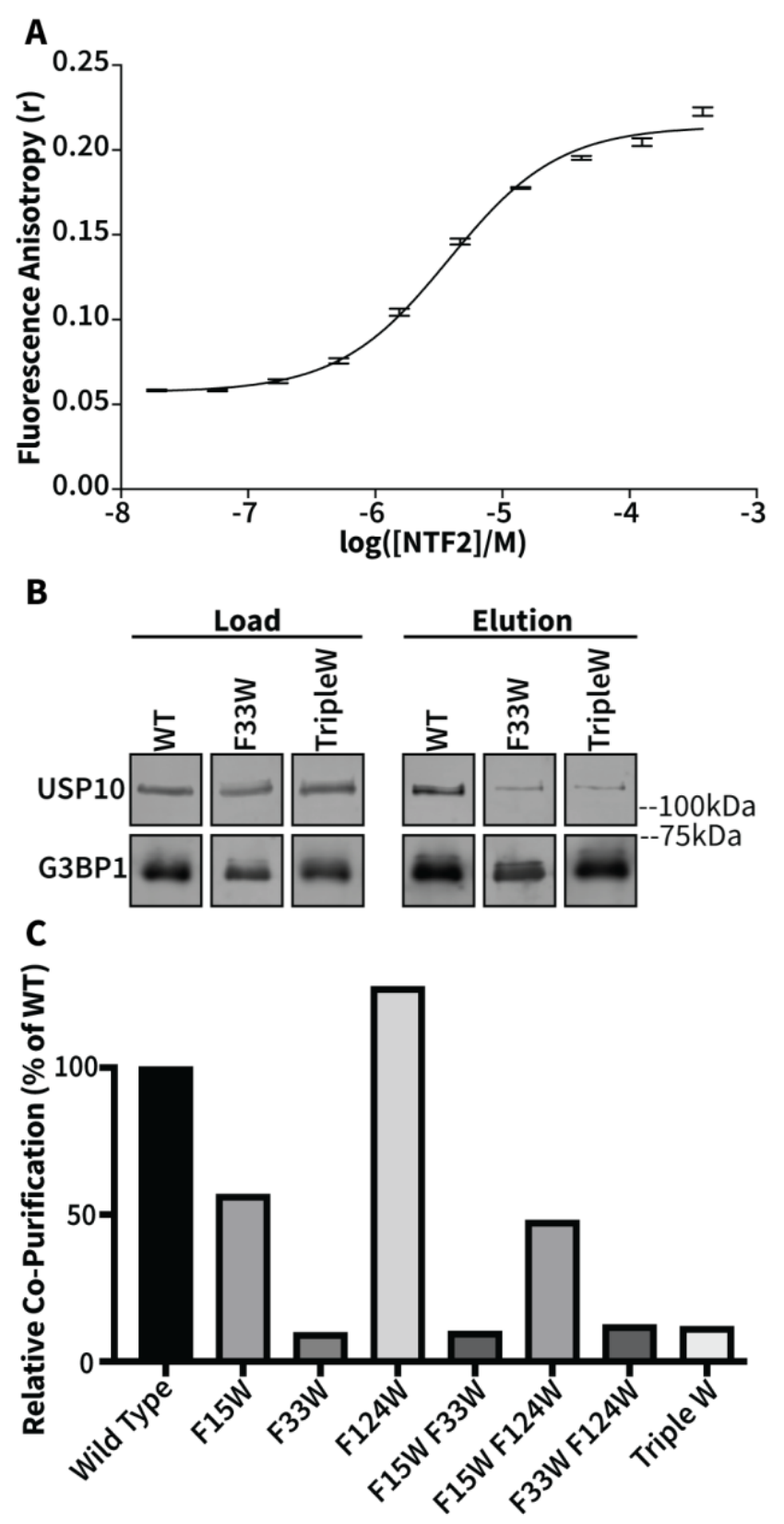

Figure 1. Individual equilibrium dissociation constants and co-purification of G3BP1:USP10 interactions. (A) An NTF2:USP10 binding isotherm was determined from fluorescence polarization assays. Fluorescence anisotropy data were collected using increasing concentrations of G3BP1 NTF2 in the presence of $30 \mathrm{nM}$ reporter peptide derived from the USP10 FGDF binding motif (Fluorescein-aminohexanoic acid[ $\left.F^{\star}\right]$-YIFGDFSP). Data are shown as mean \pm standard deviation of $n=3$ experiments. Average values were fit using a non-linear least-squares algorithm to determine $K_{\mathrm{D}}$. (B) Expi293 cells were transfected with plasmids expressing His-G3BP1 WT or mutant constructs, and lysates were subjected to affinity purification to recover His-tagged G3BP1. Equal fractions of load (left) and eluates (right) were run on SDS-PAGE followed by immunoblotting with anti-G3BP1 (bottom) and anti-USP10 (top) antibodies. (C) Immunoblots were analyzed via Image Studio, and mutants were compared to WT. Data represent the mean of $n=2$ experiments. 


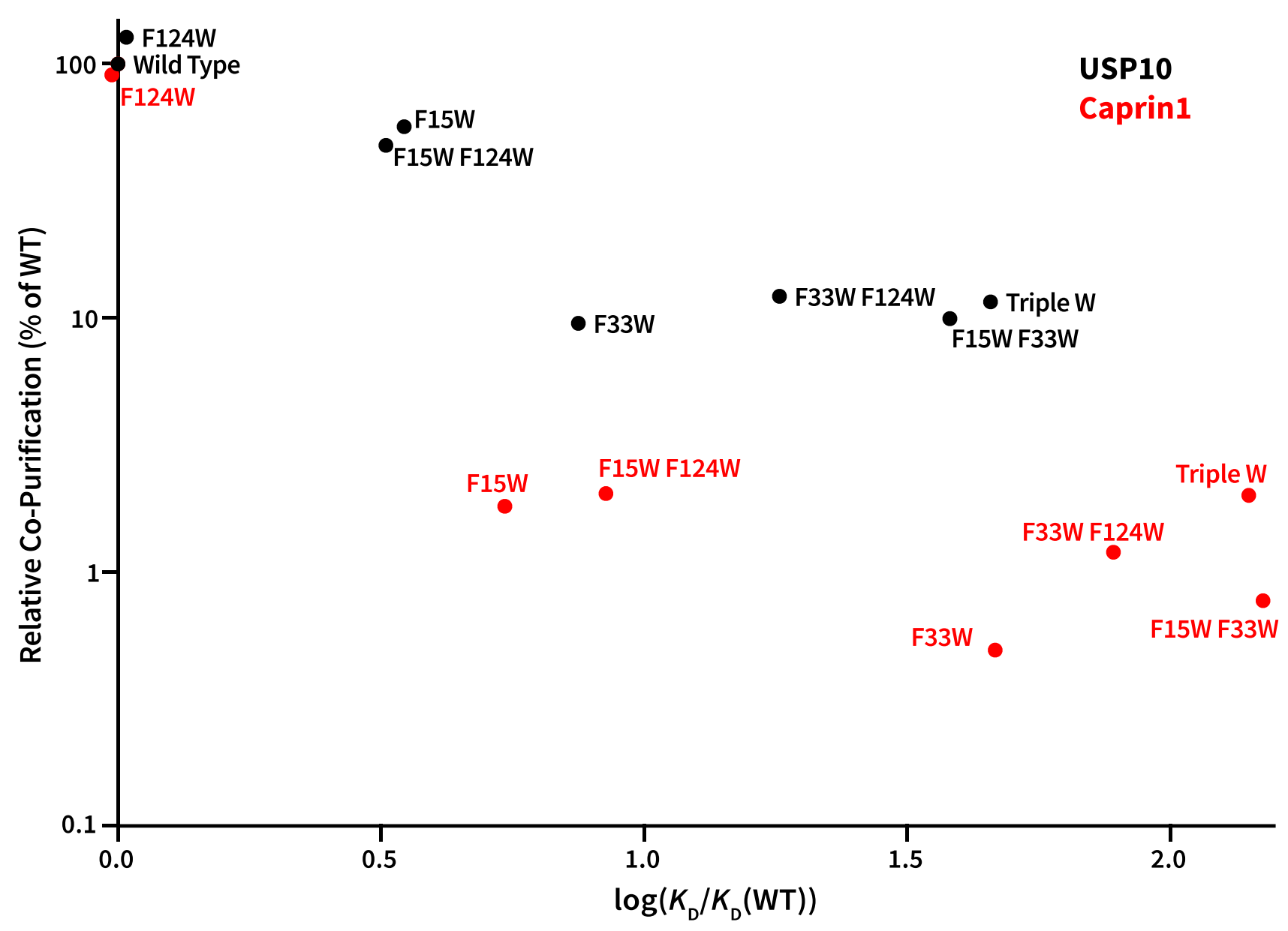

Figure 2. Comparison of relative co-purification of endogenous USP10 or Caprin1 versus corresponding equilibrium dissociation constants for G3BP1 mutants. Fulllength His-G3BP1constructs were overexpressed and affinity purified, and relative copurification percentages were calculated as described in Figures 1 and 3 . Equilibrium dissociation constants $\left(K_{\mathrm{D}}\right)$ were calculated by fluorescence polarization assays using G3BP1 NTF2 (1-139) and USP10 or Caprin1 reporter peptides. Each point represents the relative co-purification percentage and $K_{D}$ for a G3BP1mutant:USP10 (black) or G3BP1 mutant:Caprin1 (red) interaction. See also Figures 1 and 3. 

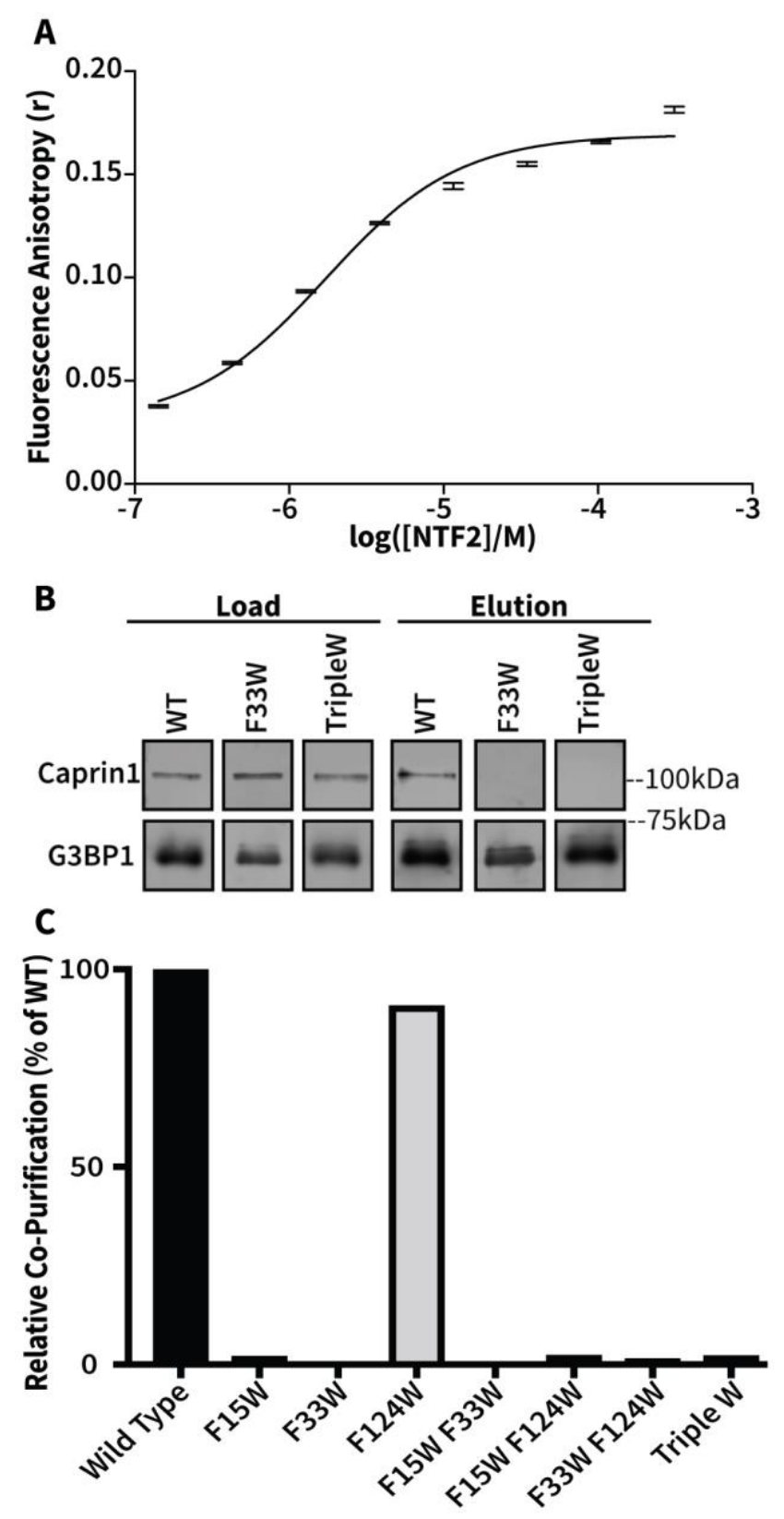

Figure 3. Individual equilibrium dissociation constants and co-purification of G3BP1:Caprin1 interactions. (A) An NTF2:Caprin1 binding isotherm was determined from fluorescence polarization assays. Fluorescence anisotropy data were collected, reported, and fit as described in Figure 1, using increasing concentrations of G3BP1 NTF2 in the presence of $30 \mathrm{nM}$ reporter peptide derived from the Caprin1 binding motif ( $F^{*}$ YNFIQDSMLDFE). Data represent the mean \pm standard deviation of $n=3$ experiments. (B) Expi293 cells were transfected with plasmids expressing His-G3BP1, WT or mutant, and lysates were subjected to affinity purification. Equal fractions of load (left) and eluates (right) were run on SDS-PAGE followed by immunoblotting with anti-G3BP1(bottom) and antiCaprin1 (top) antibodies. (C) Immunoblots were analyzed via Image Studio and mutants were compared to WT. Data represent the mean of $n=2$ experiments. 


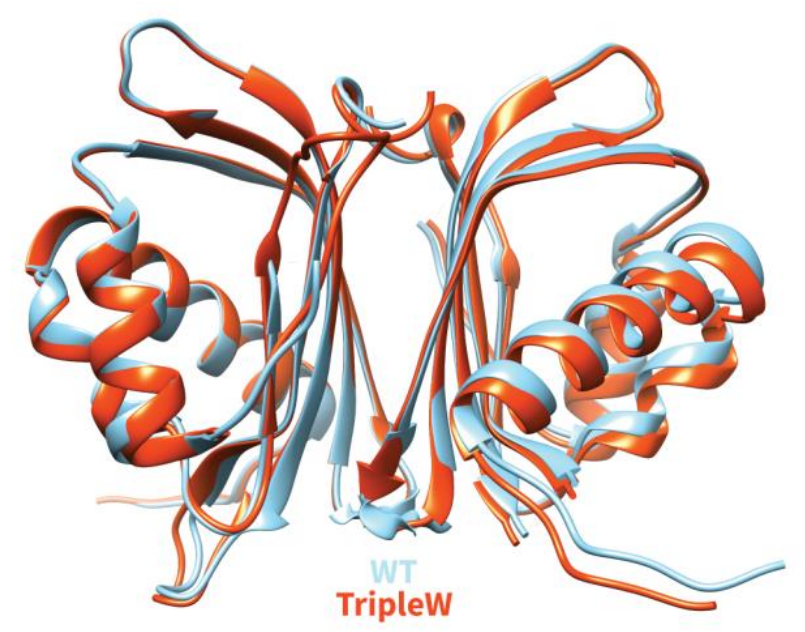

Figure 4. Structural conservation of the TripleW mutation. A cartoon representation of human G3BP1 NTF2-like domain TripleW (rust) is shown superimposed on G3BP1 NTF2like domain WT (sky blue) (PDB ID 4FCJ), with all-atom calculated rmsd of $0.57 \AA$. 
A

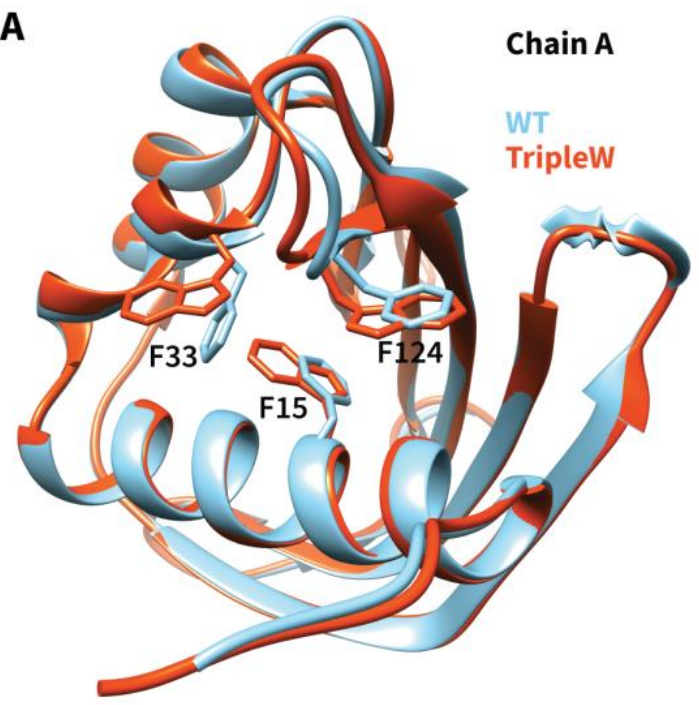

C

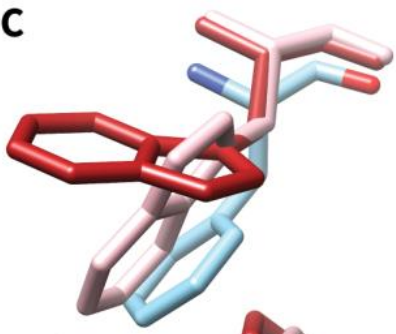

F33
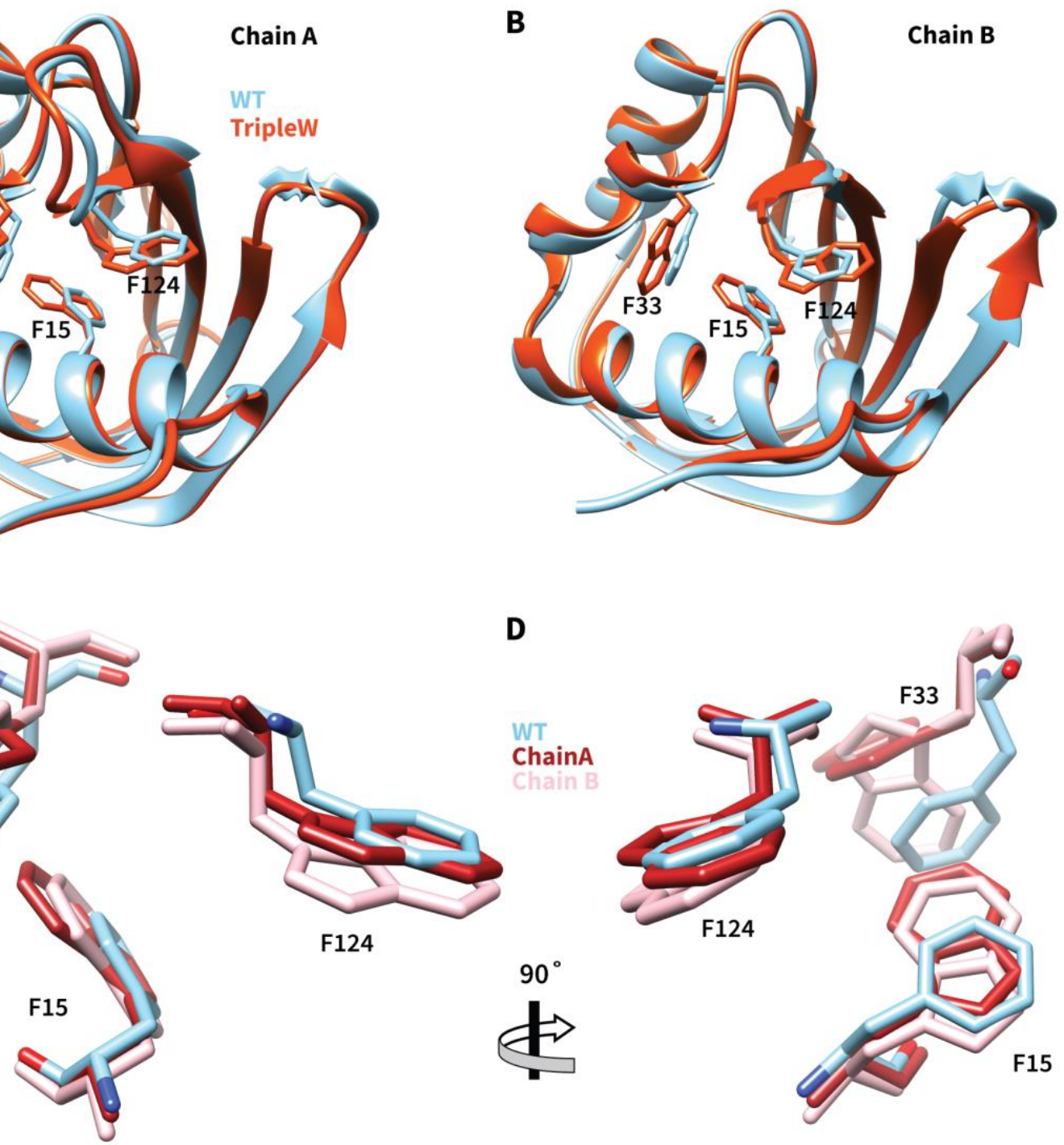

D

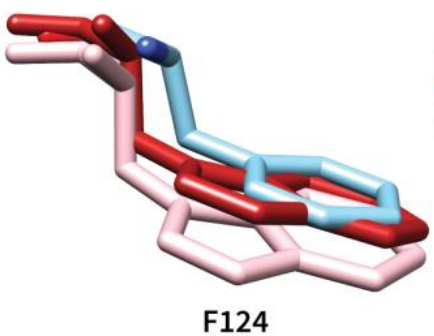

WT

ChainA
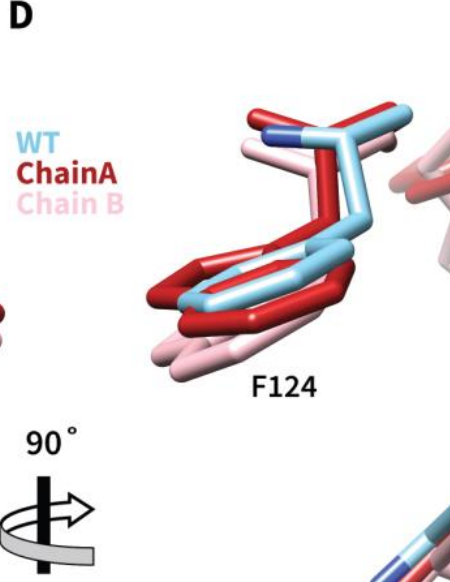

F124

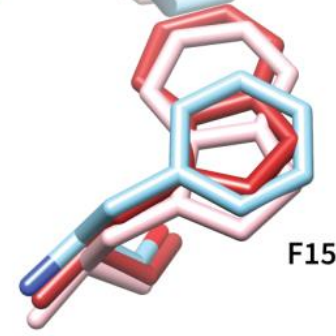

Figure 5. Stereochemical basis of Trp mutations in the NTF2 binding cleft. Cartoon representations of G3BP1 NTF2 Chain A (A) and Chain B (B) are shown, with TripleW (rust) superimposed onto WT (sky blue) (PDB ID 4FCJ). (C,D) Close-up stick-figure models show the three phenylalanine residues that are mutated to tryptophan in the TripleW structure. TripleW Chain A: red, TripleW Chain B: pink, WT Chain A: sky blue. 
A

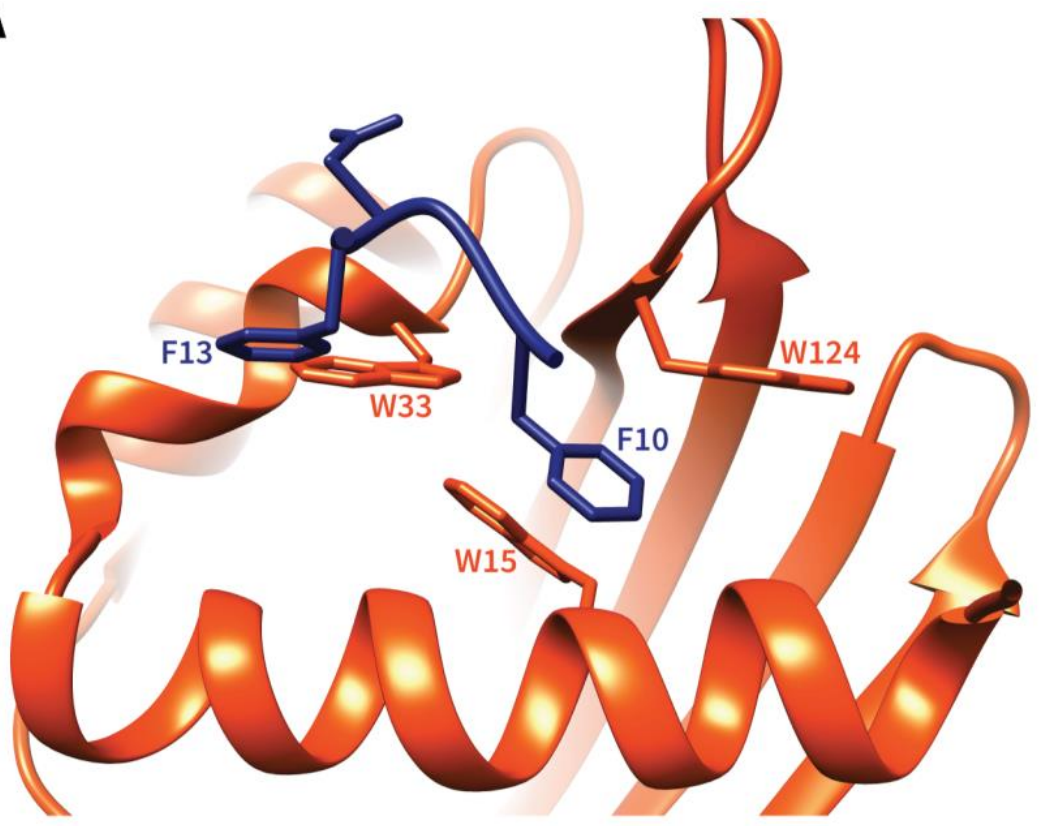

D

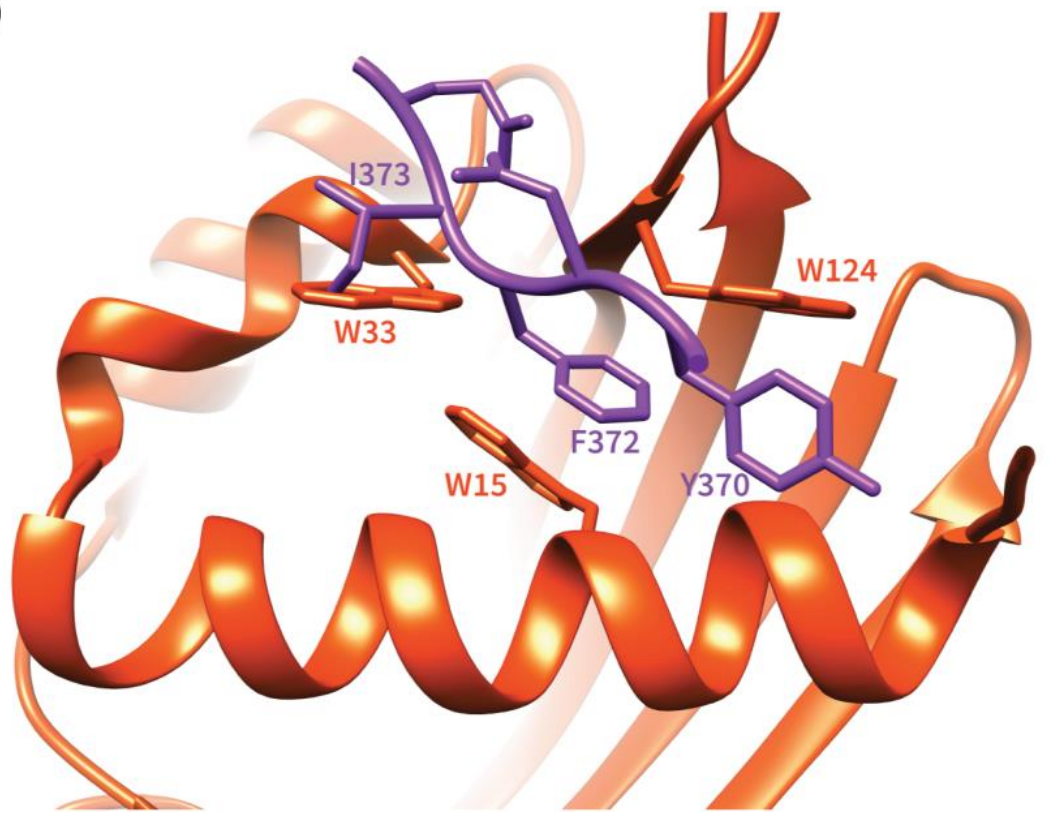

B
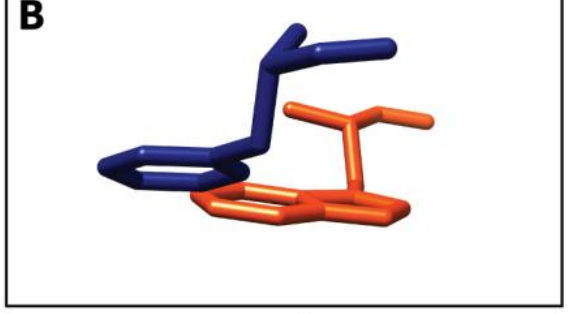

$90^{\circ}$
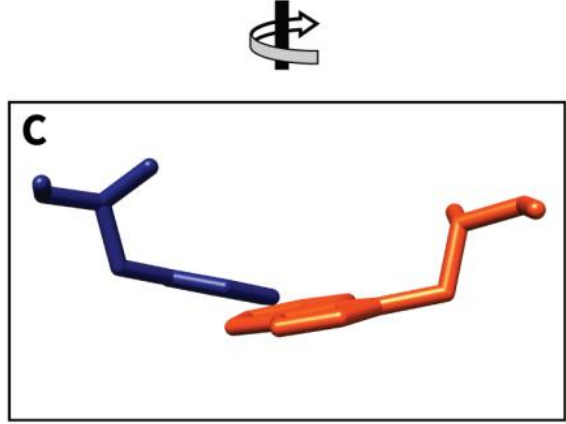

$\mathbf{E}$
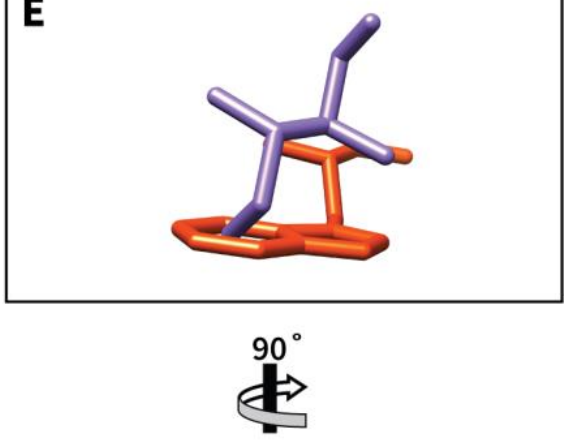

$\mathbf{F}$

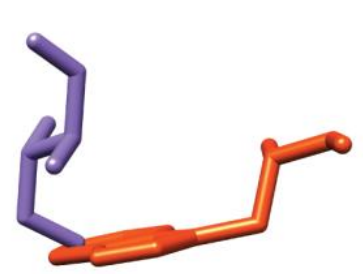

Figure 6. Impact of G3BP1 F33W mutation on USP10 and Caprin1 peptide binding. A cartoon representation of G3BP1 NTF2 Triple W is shown superimposed with the FGDF (A) and YNFIQD peptides (D) from the corresponding WT structures. The NTF2 TripleW structure was aligned with G3BP1 NTF2 WT co-crystal structures, and then the WT NTF2 domain was omitted from the figure. $(B, C)$ Isolated orthogonal views of the side-chain clash between G3BP1 NTF2 W33 (rust) and USP10 F13 (navy). (E, F) Isolated orthogonal views of the side-chain clash between G3BP1 NTF2 W33 (rust) and Caprin1 1373 (purple). The following co-crystal structures were used: $(A, B, C)$ Co-crystal structure of G3BP1 NTF2 WT with USP10 FGDF motif peptide (PDB ID 5DRV), (D, E, F) Co-crystal structure of G3BP1 NTF2 WT with Caprin1 YNFIQD motif peptide (PDB ID 6TA7). 
A
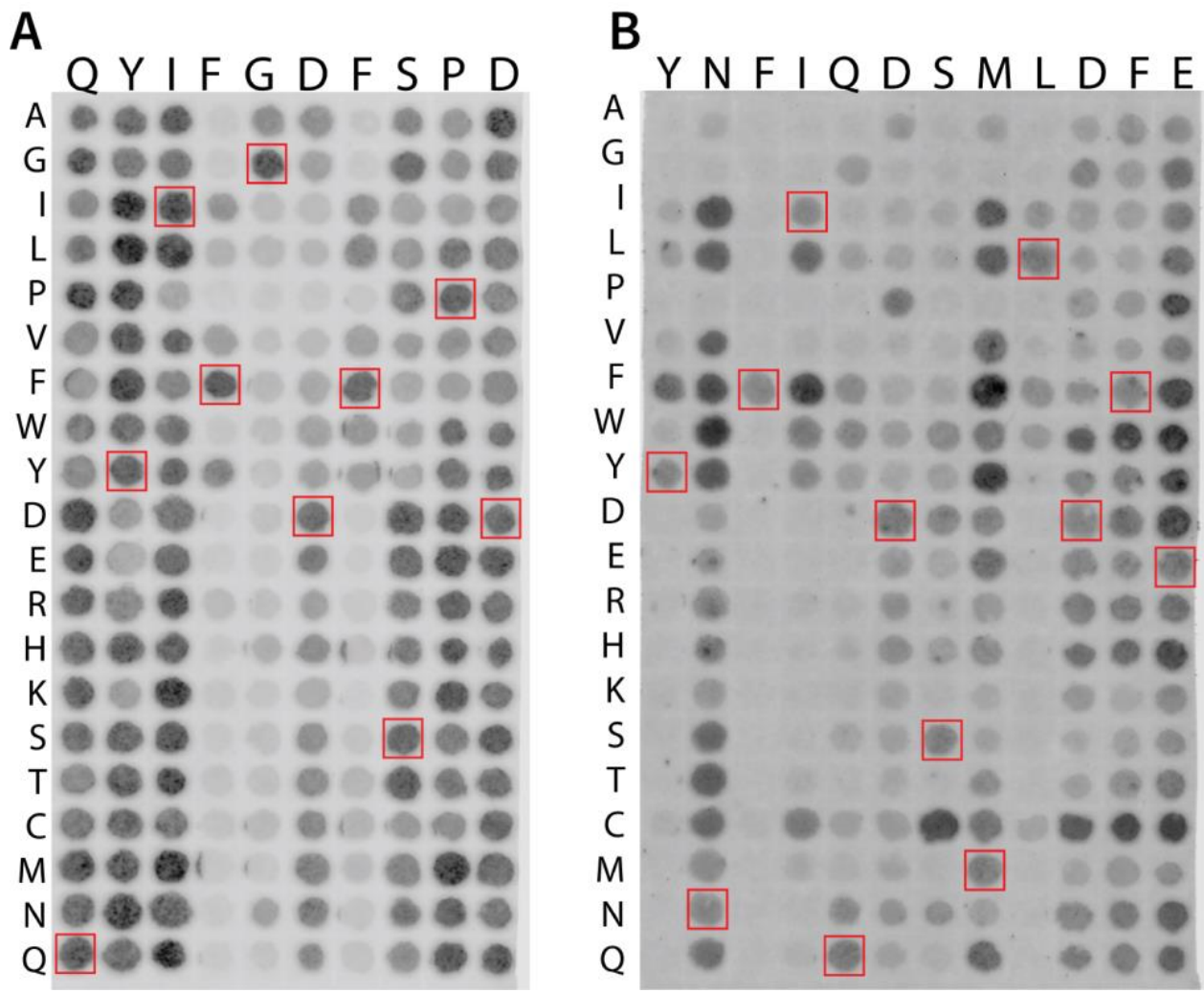

Figure 7. Binding of G3BP1 NTF2 to substituted USP10 and Caprin1 peptides. (A) Peptide cellulose SPOT array with single amino-acid substitutions in the USP10 binding peptide. Each spot contains the native USP10 peptide (HSPQYIFGDFSPDEFNQFFV) with a single amino acid in the native sequence (shown in the top row for bold residues in sequence) being replaced by the specific amino acid described by the amino-acid code in the left column. (B) Peptide cellulose SPOT array with single amino-acid substitutions in the Caprin1 binding peptide. Each spot contains the native Caprin1 peptide (QGPYNFIQDSMLDFENQTLD) with a single amino acid in the native sequence (shown in the top row for bold residues in sequence) being replaced by the specific amino acid described by the amino-acid code in the left column. Red boxes highlight peptides that match the native sequence. Darkness represents bound G3PB1 NTF2 protein as detected via antibody staining protocol. 


\begin{tabular}{|c|c|c|}
\hline \multirow{2}{*}{$\begin{array}{l}\text { NTF2 Construct } \\
\text { WT }\end{array}$} & \multicolumn{2}{|c|}{$K_{\mathrm{D}}(\mu \mathrm{M})$} \\
\hline & 3.8 & \pm 0.5 \\
\hline F15W & 13.2 & $\pm \quad 0.9$ \\
\hline F33W & 28.4 & $\pm \quad 1.1$ \\
\hline F124W & 3.9 & $\pm \quad 0.5$ \\
\hline $\mathrm{F} 15 / 33 \mathrm{~W}$ & 144.2 & $\pm \quad 3.9$ \\
\hline $\mathrm{F} 15 / 124 \mathrm{~W}$ & 12.2 & $\pm \quad 0.8$ \\
\hline $\mathrm{F} 33 / 124 \mathrm{~W}$ & 68.4 & \pm 8.4 \\
\hline TripleW & 172.3 & $\begin{array}{l} \pm \\
\pm\end{array}$ \\
\hline \multicolumn{3}{|c|}{$\begin{array}{l}K_{\mathrm{D}} \text { values were derived by non-lineal } \\
\text { least-squares fitting of the mean and } \\
\text { standard deviation values from three } \\
\text { independent FP titrations (see } \\
\text { Supplementary Figure 2). }\end{array}$} \\
\hline
\end{tabular}




\section{Table 2. Caprin1: G3BP1-NTF2 Affinities}

\begin{tabular}{lrll} 
NTF2 Construct & \multicolumn{3}{c}{$K_{\mathrm{D}}(\mu \mathrm{M})$} \\
\hline WT & 1.7 & \pm & 0.5 \\
F15W & 9.3 & \pm & 1.7 \\
F33W & 79.3 & \pm & 5.5 \\
F124W & 1.7 & \pm & 0.4 \\
F15/33W & 256.1 & \pm & 9.0 \\
F15/124W & 14.4 & \pm & 4.0 \\
F33/124W & 133.0 & \pm & 10 \\
TripleW & 240 & \pm & 13 \\
\hline KD values were estimated as described in \\
Table 1 (see Supplementary Figure 3).
\end{tabular}




\section{Table 3. Affinity of NTF2 S38 Mutants for USP10 and Caprin1}

\begin{tabular}{|c|c|c|c|c|c|c|}
\hline Mutant & \multicolumn{3}{|c|}{$K_{\mathrm{D}}(\mathrm{USP} 10)(\mu \mathrm{M})$} & \multicolumn{3}{|c|}{$K_{\mathrm{D}}($ Caprin1) $(\mu \mathrm{M})$} \\
\hline S38A & 1.3 & \pm & 0.3 & 7.3 & \pm & 1.5 \\
\hline S38G & 1.6 & \pm & 0.3 & 10.5 & \pm & 2.0 \\
\hline S38T & 9.0 & \pm & 0.4 & 11.8 & \pm & 0.4 \\
\hline
\end{tabular}

$K_{\mathrm{D}}$ values were estimated as described in Table 1 (see Supplementary Figures $2 \& 3$ ). 
Table 4. Crystal Structure Statistics

\begin{tabular}{|c|c|}
\hline Data collection \& reduction & \\
\hline Beamline & NSLS-II 17-ID-1 \\
\hline Wavelength $(\AA)$ & 0.92011 \\
\hline Space Group & $P 2{ }_{1}{ }_{1}{ }_{1}$ \\
\hline Unit cell parameters: & \\
\hline$a, b, c(\AA)$ & $42.65,71.67,87.73$ \\
\hline$\alpha, \beta, \gamma\left({ }^{\circ}\right)$ & $90,90,90$ \\
\hline Resolution ${ }^{\mathrm{a}}(\AA)$ & $19.91-2.36(2.5-2.36)$ \\
\hline$R_{\text {meas }}{ }^{\mathrm{b}}(\%)$ & $36.6(231.4)$ \\
\hline $\mathrm{CC}_{1 / 2}^{\mathrm{c}}(\%)$ & $99.1(34.9)$ \\
\hline $1 / \sigma_{\mathrm{I}}$ & $7.02(1.03)$ \\
\hline Completeness & $99.7(99.9)$ \\
\hline Redundancy & $6.4(6.0)$ \\
\hline Refinement & \\
\hline Total number of reflections & $11543(1793)$ \\
\hline Reflections in the test set & 580 \\
\hline $\mathrm{R}_{\text {work }} \mathrm{d} / \mathrm{R}_{\text {free }}{ }^{\mathrm{e}}(\%)$ & 22.2/27.1 \\
\hline Number of atoms: & \\
\hline Protein & 2204 \\
\hline Water & 17 \\
\hline Ramachandran $\operatorname{plot}^{\dagger}(\%)$ & $95.75 / 4.25 / 0$ \\
\hline RMSD bond length $(\AA)$ & 0.01 \\
\hline RMSD bond angle $\left({ }^{\circ}\right)$ & 1.07 \\
\hline PDB ID & $7 S 17$ \\
\hline
\end{tabular}

aValues in parentheses correspond to the highest resolution shell.

${ }^{\mathrm{b}} R_{\text {meas: }}$ : the redundancy independent R-factor, described in Diederichs and Karplus (1997); Nat.

Struct. Biol. 4, 269-275.

${ }^{\circ} \mathrm{CC}_{1 / 2}$ : the percentage of correlation between intensities from random half-datasets, described in detail in Karplus and Diederichs (2012); Science 336, 1030-1033.

${ }^{\mathrm{d}} R_{\text {work }}=\Sigma_{h}\left|F_{\text {obs }}(h)-F_{\text {calc }}(h)\right| / \Sigma_{h} F_{\text {obs }}(h), h \in\{$ working set $\}$.

${ }^{\mathrm{e}} R_{\text {free }}=\Sigma_{h} \mid F_{\text {obs }}(h)-F_{\text {calc }}(h) / / \Sigma_{h} F_{\text {obs }}(h), h \in\{$ test set $\}$.

${ }^{\mathrm{t}}$ Favored/allowed/outliers. 